\title{
Principles of co-axial illumination for photochemical reactions: Part 1. Model development
}

\author{
Glen Meir ${ }^{1}$, M. Enis Leblebici ${ }^{2}$, Senne Fransen ${ }^{1}$, Simon Kuhn $^{1}$, Tom Van Gerven ${ }^{1 *}$ \\ ${ }^{1}$ KU Leuven, Department of Chemical Engineering, Celestijnenlaan 200F, B-3001 Heverlee, Belgium \\ ${ }^{2}$ KU Leuven Lab4U, Faculty of Industrial Engineering, Agoralaan Building B, B-3590 Diepenbeek, \\ Belgium \\ *Corresponding author. Email address: tom.vangerven@kuleuven.be
}

\section{Compliance with ethics guidelines}

Glen Meir, Mumin Enis Leblebici, Senne Fransen, Simon Kuhn, and Tom Van Gerven declare that they have no conflict of interest or financial conflicts to disclose.

\section{ABSTRACT}

Photochemical reactors with conventional homogeneous illumination suffer from a light efficiency problem, which is inherent to their design: Dark zones arise near the reagent-rich inlet whereas the reagent depleted outlet is over-illuminated. Any attempt to mitigate dark zones at the inlet will only increase photon losses further downstream. This study reports the principles and model equations for coand counter-current illumination in photochemical reactors, along with an optimization study to determine the most efficient and productive operating point. This work proves that the use of co- and counter-current illuminated reactors increases the energy efficiency while easing scalability by implementing larger path lengths, without altering the reactor's geometry. We report a simple model to determine the conversion obtained by such novel illumination techniques and compare it to the current state-of-the-art. Two nondimensional groups where derived that describe all possible reactor configurations, these are the initial absorbance $(A)$ and the quantum photon balance $(\rho \phi)$. Variation of both parameters leads for non-

This article has been accepted for publication and undergone full peer review but has not been through the copyediting, typesetting, pagination and proofreading process, which may lead to differences between this version and the Version of Record. Please cite this article as doi: 10.1002/amp2.10044 
competitive photochemical reactions to an optimal point for the current state-of-the-art as well as the novel co-axial illumination. Ultimately, we recommend the use of an initial absorbance value $\left(A_{A}\right)$ of at least 1 , and a photon quantum balance $(\rho \phi)$ equal to 1 to introduce sufficient light and enable near complete absorption of light.

Keywords: photochemistry, photoreactors, co-axial illumination, modeling, model optimization, reactor design

\section{Introduction}

In general, the use of photochemistry has advantages compared to traditional thermochemical ways, such as higher selectivities, high conversions and mild process conditions. ${ }^{1,2}$ Photochemistry enables multi-step syntheses by providing reaction pathways, inaccessible by traditional thermochemical ways. ${ }^{2,3}$ In addition, downstream purification regarding excess reagents is avoided as the incident photons are traceless reagents. ${ }^{2,3}$ It is regarded as one of the more promising routes to achieve a more sustainable chemical production. ${ }^{1,4}$

However, despite these advantages, industrial applications of photochemistry are very limited. Current industrial reactors have numerous limitations such as: uncontrolled coupling between hydrodynamics, light, mass transfer and photochemical kinetics. ${ }^{3}$ I.e. the incident light flux density can only be as high as the photochemical kinetics or local mixing (hydrodynamics) permit, increasing the light flux beyond this point often leads to decreases in efficiency and in some cases the formation of side products. This uncontrolled coupling results in a lower selectivity and yield compared to lab scale reactors. At the present time, commercial photochemical processes are only interesting when a cheap electricity sources are available. Alternatively, energy efficiency improvements can further be achieved by reactor design. ${ }^{5}$ For example, some of these energy related problems are typical to outdated immersion-type reactor operations in semi-batch (e.g. artemisinin production) or batch (e.g. Vitamin D production, photohalogenation). ${ }^{5}$ The successful transition to continuous reactors is demonstrated for an industrial scale photoredox reactor 
developed by Merck. ${ }^{5}$ It is claimed that continuous tubular reactors allow higher conversion and selectivity due to a larger surface-to-volume ratio, lower residence times, and minimization of side reactions. $^{2-4}$ The Merck process was further intensified by the use of efficient light emitting diodes (LEDs). Nonetheless, the overall energy efficiency problem remains, making industrial photochemistry less applicable in the field of chemical synthesis. ${ }^{5}$

Recent developments in chemical reactor designs focus on the micro- and mesoscale reactor size, illuminated by energy-efficient LEDs. These geometries are excellent for overcoming mass transfer limitations in the reactor. These reactors are produced in different forms, ranging from coiled tubes around mercury lamps to plates with microchannels cut-out, illuminated from either side by LEDs. ${ }^{2,4,6}$ Inherent to their design, these reactors typically operate at a very high light to reagent balance, leading to outstanding space-time yields. Yet, many photons are lost so that the energy efficiency is poor and the associated energy cost is high. ${ }^{7,8}$

It is clear that to these small geometries waste photons severely, restricting their implementation in industry. This can be attributed to the very short photon path lengths in the reactor that results in limited, but homogeneous light absorption. ${ }^{2,4}$ Simply increasing the diameter of the mesoscale reactor does not solve these issues as larger geometries dark zones ${ }^{9}$ are formed while over-illuminated zones ${ }^{2}$ are not avoided. Both dark and over-illuminated zones are unwanted in these reactors as they respectively lead to reduced overall reaction rate and the increased energy losses..$^{2-4}$

The current challenge in photochemical reactor design for homogeneous reactions is twofold: the first challenge involves the construction and operation of an industrially competitive reactor in terms of light source position and light absorption. ${ }^{5}$ The second challenge involves the elimination of dark and overilluminated zones reducing the reactor's performance, which is challenging with current illumination techniques. ${ }^{7}$ 
In order to solve both challenges in photo-flow chemistry, the energetic inefficiency should be addressed without losing the advantages of flow chemistry. This results in a reactor geometry to be overhauled, taking the optimal light path into account, which uses the majority of the light without the creation of dark zones in the reactor. ${ }^{7,9}$ The novelty of this work is to utilize the principles of cross-, co- and counter-flow, typically found in mass and heat transfer operations. These principles are wildly utilized in distillation towers and solvent extraction cascades, counter-current has been proven to be the most efficient. This approach entails a paradigm shift: the reactor is run using a non-uniform light intensity profile to benefit the reaction, rather than operating in a homogeneously illuminated regime, see Figure 1. Soares and Padoin performed an analysis to check the more efficient illumination scheme for heterogeneous photocatalysis using $\mathrm{TiO}_{2}$ and found that back side illumination, similar to counter-current illumination was more optimal. ${ }^{10}$

The objective of this work is to investigate co-axial illumination techniques as well as the 'state-of-the-art' cross current technique by means of a mathematical model for an homogeneous photochemical reaction. An optimization study focusing on the ideal size for the specific input parameters and efficiency of the reactor is included. The overall goal is to keep the model simple, but sufficiently accurate for both lab and industrial scale applications. Future work includes the validation of this novel co-axial illumination. Concretely, the synthesis of phenylacetic acid starting from 2-chloroacetophenone in $90 \%$ acetone/water with propylene oxide as HCl-scavenger as a model reaction is described in a paired paper. ${ }^{11-13}$

\section{Model framework and derivation}

\subsection{Photochemical reactor framework}

In this section, we present equations capable of predicting reagent(s) conversion in a photochemical reactor as well as the utilization efficiency of the light. In particular, we consider a first order photochemical reaction 


$$
A \stackrel{h v}{\rightarrow} B
$$

with $A$ the reagent, $B$ the product and $h v$ the energy required for the photochemical transformation. This chemical system is in competition for light absorption, i.e. the reagent and product are capable of absorbing light at the same wavelength. However, in this model, it is assumed that species B is photostable, thus no degradation or reverse-reaction occurs. The modeling of photochemical reactors usually takes the mass balance, momentum balance, energy balance and radiative transport equations into account. ${ }^{14-16}$ Simplified models, as used in photochemical and actinometric studies, implicitly consider plug flow and homogeneous illumination, and these models yield a very good match to experimental results when used in micro- and millichannel reactors, and are considered in this work. ${ }^{17-21}$

In the following section, three different models are derived: cross-current, co-current, and counter-current illumination related to the relation between the light and flow direction. Cross-current illumination is the current state-of-the-art in photochemical reactor operation and will be treated first, see Figure 1. The coand counter-current illumination scheme are novel reactor concepts for which, to the author's knowledge, no theoretical analysis has been given before. Notably one example of co-axial illumination, utilizing both co- and counter at the same time, was found in literature in the context of actinometry. ${ }^{22}$ These two novel concepts, co- and counter-current illumination, follow the same theoretical analysis are both be covered under co-axial illumination.

Figure 1: Concept scheme of cross-, co- and counter-current illumination. Note the difference in photon path length by design and the photon losses for cross-current illumination near the end of the reactor.

\subsection{Model derivation}

\subsubsection{Cross-current illumination (state-of-the-art)}

Cross-current illumination is the 'standard' way of illuminating photochemical as well as photocatalytic reactors in flow. Characteristically, the flow direction is perpendicular with respect to the light direction. In most reactors, the average photon path length is relatively small by design (in the orders of $\mu \mathrm{m}$ to $\mathrm{mm}$ ) 
so that homogeneous light absorption is achieved, resulting in the absorption of only a small fraction of the introduced light. Although this leads to an approximately uniform "photon concentration", i.e. uniform local volumetric rate of energy absorption (LVREA), in the reactor, the majority of photons are wasted, resulting in a low energetic efficiency. ${ }^{2,23}$

\section{Geometry and mass balance}

The photochemical reactor in the cross-current illumination scheme and by extension the co- and countercurrent scheme is assumed to be tubular with a collimated, homogeneous light source. These conditions are related to, for example, a capillary tower reactor. In such a reactor, a tube is wound around an immersion well in which the lamp is placed. ${ }^{15}$ The model admits an effective path length, measured experimentally by actinometry. ${ }^{17}$

The mass balance in terms of the reagent A (see Eq. 1) is derived assuming plug flow. In this model, we explicitly assume plug flow, which is implicitly using in most papers and justified by experimental validation. ${ }^{17-21}$ The residence time distribution (RTD) factor will be added to the model in a further step.

The mass balance over a small volume element $(d V)$ can be described as:

$$
\mathrm{d} V \frac{\mathrm{d} c_{A}}{\mathrm{~d} t}=Q\left(c_{A, \text { in }}-c_{A, \text { out }}\right)+R_{A} \mathrm{~d} V=Q\left(d c_{A}\right)+R_{A} \mathrm{~d} V
$$

with $c_{A}\left[\mathrm{~mol} \mathrm{~m}^{-3}\right]$ the concentration of reagent, $Q\left[\mathrm{~m}^{3} \mathrm{~s}^{-1}\right]$ the flow rate, $d V\left[\mathrm{~m}^{3}\right]$ the volume of the reactor section and $R_{A}\left[\mathrm{~mol} \mathrm{~m}{ }^{-3} \mathrm{~s}^{-1}\right]$ the reaction rate. Which can be simplified to by assuming steady state and $\tau=V / Q[\mathrm{~s}]$, the residence time of the reactor:

$$
\frac{\mathrm{d} c_{A}}{\mathrm{~d} \tau}=-R_{A}
$$

These assumptions lead to a simple system in which the change of concentration of the reagent (component $\mathrm{A}$ ) is only dependent on the photochemical reaction rate. 
Figure 2: Mass and energy balance over an infinitesimal section $(\mathrm{d} V)$ of the photochemical reactor with a photochemical compound A. With $q_{\text {in }}, q_{\text {out }}, q_{\text {abs }}$ the incident, exiting and absorbed light flux respectively, $\mathrm{d} V$ an infinitesimal reactor volume, $c_{A}$ the concentration of compound $A, \phi$ the quantum yield, $Q$ the flow rate and $r_{A}$ the reaction rate.

\section{Derivation of photochemical reaction term}

In general the photochemical reaction rate is given by ${ }^{15,17,18,24}$

$$
R_{A}=\phi(\lambda) \boldsymbol{e}^{a}(\lambda)=\phi(\lambda) \frac{q_{a b s}(\lambda)}{\mathrm{d} V}
$$

with $\phi_{\lambda}\left[\right.$ mol einstein $\left.{ }^{-1}\right]$ the quantum yield, $\boldsymbol{e}^{\boldsymbol{a}}$ [einstein $\mathrm{s}^{-1} \mathrm{~m}^{-3}$ ] the wavelength dependent absorbed photon flux density, and $q_{a b s, \lambda}\left[\right.$ einstein $\mathrm{s}^{-1}$ ] the absorbed light flux in the reactor volume section $\mathrm{d} V\left[\mathrm{~m}^{3}\right]$. Note that $q_{a b s, \lambda}$ is also dependent on the wavelength and is given by

$$
q_{\text {abs }}(\lambda)=q_{0}(\lambda)-q_{\text {trans }}(\lambda)=q_{0, \lambda}\left(1-10^{-\sum_{A}^{n}\left(\varepsilon_{i}(\lambda) c_{i}\right) \ell}\right)=q_{0, \lambda}\left(1-10^{-\sum_{A}^{n} A_{i}(\lambda)}\right)
$$

With $q_{0}(\lambda)$ [einstein $\mathrm{s}^{-1}$ ] is the light flux entering the reactor, $q_{\text {trans }}(\lambda)$ [einstein $\mathrm{s}^{-1}$ ] the light flux leaving the reactor, $A_{i}(\lambda)$ is the absorbance of component $i$ in the solution at wavelength $\lambda[\mathrm{nm}]$, $\varepsilon_{i}(\lambda)\left[\mathrm{m}^{2} \mathrm{~mol}^{-1}\right]$ is the specific absorbance of component $i$ per wavelength, $c_{i}\left[\mathrm{~mol} \mathrm{~m}^{-3}\right]$ the concentration of component $i$ in solution and $\ell[\mathrm{m}]$ is the path length. In order to compensate for any competitive absorbance by components $i$ to $n$ as indicated in Equation (5), $q_{\mathrm{abs}}$ is multiplied by correction factor $\Gamma$,

$$
\Gamma=\frac{A_{A}}{\sum_{i=A}^{n} A_{i}}=\frac{\varepsilon_{A}(\lambda) c_{A}(\tau)}{\sum_{i=A}^{n}\left(\varepsilon_{i}(\lambda) c_{i}\right)}
$$

as suggested by Aillet et al. ${ }^{15}$ Ultimatly, the reaction rate follows from Equations (4), (5) and (6).

$$
R_{A}=\phi(\lambda) \frac{q_{0}(\zeta, \lambda)\left(1-10^{-\sum_{i=A}^{n}\left(\varepsilon_{i}(\lambda) c_{i}(\tau)\right) \ell}\right)}{\mathrm{d} V} \frac{\varepsilon_{A}(\lambda) c_{A}(\tau)}{\sum_{i=A}^{n}\left(\varepsilon_{i}(\lambda) c_{i}(\tau)\right)}
$$

where $q_{0}(\zeta, \lambda)$ is expressed at normalized axial position $\zeta[-]$ in relation to the axial distance in the reactor. Equation 7 is valid for all three illumination schemes. From here, the light inlet parameter changes for the three techniques and has an influence on the required formulas, i.e. path length and light influx. Therefore, the derivation is split into cross-current and co-axial from this point. 
For cross-current, $q_{0}(\zeta, \lambda)$ can also be written as $G_{0}(\zeta, \lambda)\left[\right.$ einstein $\left.\mathrm{m}^{-2} \mathrm{~s}^{-1}\right] d S\left[\mathrm{~m}^{2}\right]$ to explicit the irradiance intensity times the projected area of illumination. Equation 7 results:

$$
\frac{\mathrm{d} c_{A}}{\mathrm{~d} \tau}=-R_{A}=-\phi(\lambda) \frac{G_{0}(\zeta, \lambda) \mathrm{d} S\left(1-10^{-\sum_{i=A}^{n}\left(\varepsilon_{i}(\lambda) c_{i}(\tau)\right) \ell}\right)}{\mathrm{d} V} \frac{\varepsilon_{A}(\lambda) c_{A}(\tau)}{\sum_{i=A}^{n}\left(\varepsilon_{i}(\lambda) c_{i}(\tau)\right)}
$$

This equation is valid for an infinitesimal section with width $d x$ in axial direction of the reactor. As a result the projected area of illumination $(\mathrm{d} S=2 R \mathrm{~d} x)$ and section volume $\left(\mathrm{d} V=\pi R^{2} \mathrm{~d} x\right)$ have to be written as such, resulting in:

$$
\frac{\mathrm{d} c_{A}}{\mathrm{~d} \tau}=-\phi(\lambda) \frac{G_{0}(\zeta, \lambda) 2\left(1-10^{-\sum_{i=A}^{n}\left(\varepsilon_{i}(\lambda) c_{i}(\tau)\right) \ell}\right)}{\pi R} \frac{\varepsilon_{A}(\lambda) c_{A}(\tau)}{\sum_{i=A}^{n}\left(\varepsilon_{i}(\lambda) c_{i}(\tau)\right)}
$$

This equation is made non-dimensional by defining the following non-dimensional parameters: $X$ the conversion of component $\mathrm{A}, \iota$ inwards light flux at relative position $\zeta$ vs. average light flux $\left(G_{0}(\lambda)\right)$ and $\zeta$ the relative axial position in the reactor.

$$
\begin{gathered}
X(\zeta)=\frac{c_{A, 0}-c_{A}}{c_{A, 0}} \\
\iota(\zeta, \lambda)=\frac{G_{0}(\zeta, \lambda)}{G_{0}(\lambda)} \\
\zeta=\frac{x}{L} \\
\tau=\frac{V}{Q}=\frac{A L \zeta}{Q}
\end{gathered}
$$

Applying these non-dimensional quantities in Equation (9 results in:

$$
\begin{aligned}
& \frac{\mathrm{d} X(\zeta)}{\mathrm{d} \zeta} \\
& =\iota(\zeta, \lambda) \phi(\lambda) \frac{G_{0}(\lambda) 2 R L}{Q c_{A, 0}}(1 \\
& \left.-10^{-\left(\varepsilon_{A}(\lambda) c_{A, 0} \ell(1-X(\zeta))+\ell \sum_{n}\left(\varepsilon_{i}(\lambda) c_{i}\right)\right)}\right) \frac{\varepsilon_{A}(\lambda) c_{A, 0}(1-X(\zeta))}{\varepsilon_{A}(\lambda) c_{0, A}(1-X(\zeta))+\sum_{i=B}^{n}\left(\varepsilon_{i}(\lambda) c_{i}\right)}
\end{aligned}
$$


It must be noted that the concentration of the $n$ additional species is kept variable, but can be related directly to the conversion $(X)$ of reagent $A$ if constant reaction selectivity is observed. In Eq. 11, two nondimensional groups can be observed. The first $\left(\rho_{A}\right)$ takes into account the balance of light versus reagent and the second is commonly known as the absorbance $(A)$.

$$
\begin{gathered}
\rho_{A}(\lambda)=\frac{G_{0}(\lambda) 2 R L}{Q c_{A, 0}} \\
A_{i}(\lambda)=\varepsilon_{i, \lambda} c_{i, 0} \ell
\end{gathered}
$$

With the implementation of these groups, the following equation is obtained:

$$
\frac{\mathrm{d} X(\zeta)}{\mathrm{d} \zeta}=\iota(\zeta, \lambda) \phi(\lambda) \rho_{A}(\lambda)\left(1-10^{-\left(A_{A}(\lambda)(1-X(\zeta))+A_{i}(\lambda)\right)}\right) \frac{A_{A}(\lambda)(1-X(\zeta))}{A_{A}(\lambda)(1-X(\zeta))+A_{i}(\lambda)}
$$

Practically, Eq. 13 is applicable for cross-current illumination and is typically solved for $X(0)=0$ as initial condition, which corresponds to no product being present in the inlet solution. An overview of the in- and output in non-dimensional parameters, as well as the boundary conditions is shown in Figure 3.

Figure 3: Schematic overview showing the influx and outflux, the boundary conditions for cross-current illumination using a photochemical compound A. With $q$ the light flux, $\iota$ the non-dimensional light flux, $\zeta$ the non-dimensional axial position, $\mathrm{d} V$ an infinitesimal reactor volume, $Q$ the flow rate, $c_{A}$ the concentration of compound $A, X$ the conversion, $r_{A}$ the reaction rate, and $\phi$ the quantum yield. These symbols are the same as in Eq. 13.

In Eq. 13, it is important to indicate that $\iota_{\zeta, \lambda}$ is still present, indicating the dependence of $\iota$ (light fraction remaining) to the axial coordinate of the reactor. For homogeneous illumination $\iota=1$ and is furthermore useful when a variable light inlet is applied, i.e. the light output of the LEDs is decreased with respect to axial reactor position. An extension to this model is found in the Supplementary Information, see section S1, including wavelength distribution, residence time distribution, and the influence of reflective reactor material. 


\subsubsection{Co- and counter-current illumination (co-axial)}

Co- and counter-current illumination differs from cross-current illumination in terms of the path length of the light and axis of light propagation. As reagent flows axially, parallel to the direction of light, a new problem is introduced: it can no longer be assumed that concentration along the light propagation direction is constant. This results in a concentration and light flux change along the axis of propagation $(\zeta)$. Both problems are coupled and must solved simultaneously. An overview in non-dimensional parameters is found in Figure 4.

The co- and counter-current equations are derived for plug flow, but will later be extended to cases where the RTD is known. The applicability of these RTD in co- and counter-current modeling can be limited when using non-collimated LEDs, as these LEDs enhance the radial distribution of the light.

Co-axial illumination differs from cross-current starting from Eq. 7. An absorbance gradient is present along the path length and is therefore discretized in $\mathrm{d} x$ as

$$
\frac{\mathrm{d} c_{A}}{\mathrm{~d} \tau}=-\phi(\lambda) \frac{q_{0}(\zeta, \lambda)\left(1-10^{\left.-\sum_{A}^{n}\left(\varepsilon_{i}(\lambda) c_{i}\right) \mathrm{d} x\right)}\right.}{\mathrm{d} V} \frac{\varepsilon_{A}(\lambda) c_{A}}{\sum_{i=A}^{n}\left(\varepsilon_{i}(\lambda) c_{i}\right)}
$$

The volume $(\mathrm{d} V)$ is written in terms of its axial cross-sectional area $(S)$ and infinitesimal reactor length $(\mathrm{d} x)$ :

$$
\frac{\mathrm{d} c_{A}}{\mathrm{~d} \tau}=-\phi(\lambda) \frac{q_{0}(\zeta, \lambda)\left(1-10^{\left.-\sum_{A}^{n}\left(\varepsilon_{i}(\lambda) c_{i}\right) \mathrm{d} x\right)}\right.}{S \mathrm{~d} x} \frac{\varepsilon_{A}(\lambda) c_{A}}{\sum_{i=A}^{n}\left(\varepsilon_{i}(\lambda) c_{i}\right)}
$$

Since the absorbance over $d x$ is infinitesimal, a Taylor expansion (around 0) of the exponent can be carried out

$$
\frac{\mathrm{d} c_{A}}{\mathrm{~d} \tau}=-\phi(\lambda) \frac{q_{0}(\zeta, \lambda) \ln (10) \sum_{A}^{n}\left(\varepsilon_{i}(\lambda) c_{i}\right) \mathrm{d} x}{S \mathrm{~d} x} \frac{\varepsilon_{A}(\lambda) c_{A}}{\sum_{i=A}^{n}\left(\varepsilon_{i}(\lambda) c_{i}\right)}
$$

The same non-dimensional terms as in Eq. 10 can be introduced. Note that for $\iota \zeta, \lambda$ is used, which has the same meaning: $\iota(\zeta, \lambda)=\frac{q_{0}(\zeta, \lambda)}{q_{0}(\lambda)}$. Implementation leads to 


$$
\begin{aligned}
& \frac{\mathrm{d} X(\zeta)}{\mathrm{d} \zeta}=\iota(\zeta, \lambda) \phi(\lambda) \frac{q_{0}(\lambda)}{Q c_{A, 0}} \ln (10)\left(\varepsilon_{A}(\lambda) c_{A, 0} L(1-X(\zeta))\right. \\
& \left.+L \sum_{n}\left(\varepsilon_{i}(\lambda) c_{i}\right)\right) \frac{\varepsilon_{A}(\lambda) c_{A, 0}(1-X(\zeta))}{\varepsilon_{A}(\lambda) c_{A, 0}(1-X(\zeta))+\sum_{n}\left(\varepsilon_{i}(\lambda) c_{i}\right)}
\end{aligned}
$$

Herein, the same non-dimensional groups as in Eq. 12 can be observed, however, note that $\rho_{A}(\lambda)$ is written in terms of total light flux $\left(q_{0}(\lambda)\right)$ rather than the incident intensity multiplied by the projected area $\left(G_{0}(\lambda) 2 R L\right)$ as used in cross-current illumination. In the end, the physical meaning is identical.

$$
\rho_{A}(\lambda)=\frac{q_{0}(\lambda)}{Q c_{A, 0}}
$$

Using these groups and simplifying the correcting term for competitive absorbance, a rather compact model is ultimately acquired:

$$
\frac{\mathrm{d} X(\zeta)}{\mathrm{d} \zeta}=\iota(\zeta, \lambda) \phi(\lambda) \rho_{A}(\lambda) \ln (10) A_{A}(\lambda)(1-X(\zeta))
$$

A graphical presentation of each flux is shown in Figures 4 and 5 for co- and counter-current, respectively.

Figure 4: Schematic overview showing the influx and outflux, the boundary conditions for co-current and countercurrent illumination using a photochemical compound A. With $q$ the light flux, $\iota$ the non-dimensional light flux, $\zeta$ the non-dimensional axial position, $\mathrm{d} V$ an infinitesimal reactor volume, $Q$ the flow rate, $c_{A}$ the concentration of compound $A, X$ the conversion, $r_{A}$ the reaction rate, and $\phi$ the quantum yield. These symbols are the same as in Eq. 19.

The implementation of wavelength distribution and residence time distribution is found in the Supplementary Information (see Section S2). Equation 19 is typically solved using $X(0)=0$, however the remaining light fraction $(\iota(\zeta, \lambda))$ at position $\zeta$ is dependent on the axial position in the reactor and is not yet expressed in an equation. The light fraction that remains after light passes through a slice of the reactor is still an unknown and can be determined by the law of Lambert-Beer. The remainder of the light can be written with respect to absorption of the reagent $A$ and other components $i$ at slice at position $x+1$ with the light passing slice at position $[\mathrm{m}]$ : 


$$
q_{0}(x+d x, \lambda)=q_{0}(x, \lambda) 10^{-A_{\lambda}}=q(x, \lambda) 10^{-\sum_{i=A}^{n}\left(\varepsilon_{i}(\lambda) c_{i}(x)\right) \mathrm{d} x}
$$

This equation can be written in differential form after the application of a Taylor expansion of the exponent (around 0) which is cut off after the first term. This absorbance is still expressed with respect to position $(x)$ :

$$
\frac{\mathrm{d} q_{0}(x, \lambda)}{\mathrm{d} x}=-q_{0}(x, \lambda) \ln (10) \sum_{i=A}^{n}\left(\varepsilon_{i}(\lambda) c_{i}(x)\right)
$$

Using the same non-dimensional parameters as found in Eq. 10, this system can be made non-dimensional

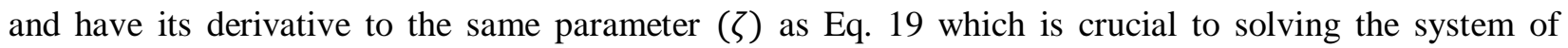
differential equations. After simplification, the following expression is obtained for co-current, this is typically solved with $\iota(0)=1$ as initial condition, i.e. $100 \%$ light inlet.

$$
\frac{\mathrm{d} \iota(\zeta, \lambda)}{\mathrm{d} \zeta}=-\iota(\zeta, \lambda) \ln (10)\left(A_{A}(\lambda)(1-X(\zeta))+\sum_{i=B}^{n} A_{i}(\lambda)\right)
$$

For counter-current, the equation differs by the sign, as shown below. This is so because the light is introduced at the end of the reactor, thus increasing the intensity from inlet to outlet. Here, the initial condition, $\iota(1)=1$ is applied. Hence, the position of this boundary condition (end of the reactor) is different from Eq. 19 which is to be solved in conjunction with Eq. 22. An iterative or numerical technique is applied to solve this system.

$$
\frac{\mathrm{d} \iota(\zeta, \lambda)}{\mathrm{d} \zeta}=\iota(\zeta, \lambda) \ln (10)\left(A_{A}(\lambda)(1-X(\zeta))+\sum_{i=B}^{n} A_{i}(\lambda)\right)
$$

For systems with polychromatic light sources, Eqs. 22 and 23 turn into an array $\left(\boldsymbol{\iota}_{\zeta, \lambda}\right)$ of differential equations spanning the range of $\lambda_{1}$ up to $\lambda_{2}$ with the discretization level based on the numerical solver.

\subsubsection{Overview of equations}

The following equations with initial conditions have to be solved to predict conversion in the reactor and the remaining light fraction. An overview in Table 1 is provided. The equations contain the correction for polychromatic light (in blue), the correction for residence time distribution (in red) and absorption of 
chemicals other than the reagent (in green). Any of these complete terms can be omitted completely if monochromatic light is used (blue part), complete plug flow (red part) and/or systems with no absorption other than the reagent (green part), respectively.

Table 1 Full equations and initial conditions (I.C.) to model cross-, co- and counter-current illumination. The equations include absorbance by various chemicals (in green), complex flow regimes via residence time distribution (in red) and polychromatic light e.g. LEDs (in blue).

\section{Solution methods}

\subsection{General solution strategy}

A reactor using plug flow and laminar will be explored as the flow boundaries. The used equations are simplified to limit the number of variables for this optimization study. The full residence time distribution is omitted, an average specific absorbance value is used and it is assumed that no competitive absorption takes place. These assumptions lead to the following equations as shown in Table 2 .

Table 2 Simplified equations and initial conditions (I.C.) used for parametric evaluation w.r.t. the use of plug flow regime, monochromatic light input and absorbance solemnly by the reagent, leading to the colored terms to be omitted from Table 1.

In these equations, two independent variables; $\rho \phi$ (quantum photon balance) and $A_{A}$ (absorbance) can be found. Variation of these two parameters with plug flow and laminar flow behaviour is performed below to investigate an optimum operating point which is useful in the design of novel reactors and to check the efficiency of current operating reactors.

MATLAB 2018b is used to solve the equations presented in Table 2. The system of coupled differential equations are implemented in a function script. Via de use of 'ode45' solver, a medium accuracy solver for non-stiff problems which is perfect for these equations, the equations are solved for $(X(\zeta), \iota(\zeta))$ between a span of [0 1] referring to the non-dimensional length of the reactor and initial conditions [ $\left[\begin{array}{ll}0 & 1\end{array}\right]$ 
for cross- and co-current illumination referring to conversion of $0 \%$ and initial light flux of $100 \%$ at the inlet of the reactor.

For counter-current a more complicated solution strategy is applied. Since the boundary conditions referring to conversion and light inlet are positioned at different $\zeta$-values, the 'standard' operating procedure as described above is not possible. Therefore the following initial conditions are applied [0 $\left.\iota_{c o, o u t}\right]$ as initial conversion of $0 \%$ and initial outward light flux, keeping in mind that the differential equation is solved from inlet to outlet of the reactor opposite to the light flux direction. Via the use of an iterative procedure using the 'f-solve'-function, the difference between $\iota_{\text {counter,out-calc }}$ $\iota_{\text {counter,out-set }}$, note that $\iota_{\text {counter,out-set }}$ equals 1 i.e. $100 \%$ and $\iota_{\text {counter,out-calc }}$ is obtained via the aforementioned solution strategy using the 'ode 45 ' solver. Via this procedure, the correct initial condition at $\iota(0)$ can be determined and solved using the aforementioned 'ode 45 ' solver, with the correct initial conditions at the inlet of the reactor.

Fully numerical solution strategies exist which can cope with boundary conditions at different positions, however, these methods are not explored here.

\subsection{Plug flow}

The developed models for cross-, co- and counter-current illumination are capable of predicting the conversion as well as the efficiency of light utilization. The optimal operation point of such a reactor is the combination of high conversion and efficient light utilization. To quantify both parameters, an efficiency benchmark is introduced

$$
\eta=X_{\text {out }}\left(\iota_{\text {in }}-\iota_{\text {out }}\right)
$$

where $\iota_{\text {out }}$ is averaged over the length of the reactor for cross-current and retained as a single value by the model for co-axial illumination. This benchmark varies between 0 for a poorly performing reactor and 1 for the most efficient reactor possible. thus high efficiency relates to high values of $\eta$ and vice-versa. This benchmark is calculated for a simplified model using monochromatic light and no absorbing components 
except for the reagent. A second benchmark is the PSTY (photochemical space time yield), commonly used to compare photochemical reactor designs. This benchmark was introduced by Leblebici et al. ${ }^{9}$ and provides a measure for the reactors performance by dividing the space time yield STY [mol s $\mathrm{m}^{-1}$ ] of the reactor by the normalised lamp power LP [W]. The lamp power will be expressed in flux of incident light [einstein $\mathrm{s}^{-1}$ ] in the reactor and will not consider the power drawn by the entire setup in this comparison, in order to only consider the illumination method. This benchmark is described by the following equation and equals 0 for a poor performing reactor and $\phi$ for the best productivity possible.

$$
\text { PSTY }=\frac{\mathrm{STY}}{\mathrm{LP}}=\frac{\frac{X_{\mathrm{out}} c_{0} V}{\tau V}}{\frac{q_{0}}{V}}=\frac{X_{\mathrm{out}} c_{0} V}{\tau q_{0}}=\frac{X_{\mathrm{out}} c_{0} V Q}{V q_{0}}=\frac{X_{\mathrm{out}}}{\rho}[-]
$$

\subsection{Laminar flow}

Typical milli-scale photochemical reactors operate at a Fo number below 1, limiting radial diffusion in the reactor. Laminar flow in these reactors is characterized by its characteristic parabolic velocity profile. There are two ways to implement this in the derived models. One option is to implement the residence time distribution, as described below ${ }^{25}$ :

$$
E_{\theta}=\frac{1}{2 \theta^{3}} \text { for } \theta \geq \frac{1}{2} \text { and } \mu_{\theta}=1
$$

With $E_{\theta}$ the response curve for the residence time distribution of a vessel, $\theta[-]$ the normalised residence time $(V / u)[-]$ and $\mu_{\theta}[-]$ the average value of the residence time distribution. Another option it to implement the velocity profile itself, as given below ${ }^{25}$ :

$$
u(r)=2 u_{\mathrm{avg}}\left(1-\frac{r^{2}}{R^{2}}\right)
$$

With $u$ the velocity profile, $u_{\mathrm{avg}}[\mathrm{m}]$ the average velocity, $r[\mathrm{~m}]$ the radial length and $R[\mathrm{~m}]$ the outer radius of the tube. In this case, as the formulas are implemented using a differential approach, the velocity profile will be used. The boundaries of this velocity profile are closed and are $\left[\begin{array}{ll}0 & 2 u_{\mathrm{avg}}\end{array}\right]$. The boundaries 
of the residence time distribution are $[0+\infty$ [ and this is computationally more challenging to integrate in the program.

The implementation of the laminar flow has a direct influence on the $\rho$ value as derived before.

$$
\rho_{A}(r, \lambda)=\frac{q_{0}(\lambda)}{(u(r) Q) c_{0, A}}=\frac{q_{0}(\lambda)}{\left(u(r) u_{a v g}\right) A c_{0, A}}
$$

When $u_{\mathrm{avg}}=1$ and $R=1$ and $r=\left[\begin{array}{ll}0 & 1\end{array}\right]$, it can be used as a normalized distribution.

$$
\rho_{A}(r, \lambda)=\frac{q_{0}(\lambda)}{2\left(1-r^{2}\right) Q c_{0, A}}=\frac{\rho_{A}(\lambda)}{2\left(1-r^{2}\right)}
$$

In order to simulate a specific chosen $\rho_{A}$ value, multiple simulations are performed, each for a specific radial position in the reactor, $r=\left[\begin{array}{ll}0 & 1\end{array}\right]$. These individual radial solutions are weighed afterwards using the mixed cup average w.r.t. conversion, with a discretization level of parameter $r$ dependent on the integration method ${ }^{26}$ :

$$
X_{\text {end,avg }}=\frac{\int_{0}^{1} X_{\text {end }}(r) u(r) 2 \pi r \mathrm{~d} r}{\int_{0}^{1} 2 \pi r d r}=\int_{0}^{1} X_{\text {end }}(r) 2\left(1-r^{2}\right) 2 r \mathrm{~d} r
$$

To determine the average light losses, an average by area is taken and implemented numerically:

$$
\iota_{\text {out }, \text { avg }}=\frac{\int_{0}^{1} \iota_{\text {out }}(r) 2 \pi r \mathrm{~d} r}{\int_{0}^{1} 2 \pi r \mathrm{~d} r}=\int_{0}^{1} \iota_{\text {out }}(r) 2 r \mathrm{~d} r
$$

With $X_{\text {end }}(r)$ and $\iota_{\text {out }}(r)$ the conversion at the outlet of the reactor and overall light losses, respectively. The average light loss is different for each illumination technique using $\rho_{r}$ as input. These expressions can be found in Table 1 for the full integrated equation and in Table 2 for the simplified equations, that are used in this parameter study. 


\section{Optimization results and discussion}

\subsection{Determination of optimal operating conditions for plug and laminar flow}

\subsubsection{Parameter study - plug flow}

The equations as reported in Table 2 are evaluated by variation of $\rho \phi$ between $0.75-1.25$ and $A$ between $0-10$. These high values of $A>2$ often do not follow the law of Lambert-Beer any more, but near-full extinction of light is present at these values, which is what is used by the model. ${ }^{27}$ The conversion, light efficiency and benchmark value is given for each illumination technique below.

\section{Cross-current}

Figure 5 Conversion (a) and light utilization efficiency (b), benchmark $\left(\mathrm{X}_{\text {out }}\left(1-\mathrm{t}_{\text {out }}\right)\right)(\mathrm{c})$ and PSTY (d) vs absorbance (A) and light/reagens ratio $(\rho \phi)$, higher values indicate higher conversion and/or higher light efficiency for cross-current with a plug flow regime. The benchmark's range is [0 1], with 1 being the most optimal.

Based on Figure 5, it can be concluded that high absorbance values are optimal towards achieving high conversions and high efficiencies. Regarding conversion, $\rho \phi>1$ can yield near-full conversion with the loss of light as a consequence. The best light efficiency is achieved at $\rho \phi<1$ at the cost of conversion, in this region the reactor performs optimal according to the PSTY. Although this analysis indicates that high conversions can be reached with decent light efficiency, in practice this is not the case. In the most common reactor geometries, these higher absorbance values are not met. Commonly, millireactor, microreactor and thin film reactors are used which are designed for homogeneous illumination, thus having low up to very low absorbance values (e.g. $<0.1) .^{2,4,5,15,18}$ If one was to operate these reactors at higher absorbances, the selectivity of the reaction might suffer. Furthermore, at an absorbance of 1 , half of the light is absorbed in the first quarter of the reactor, losing homogeneous illumination. In the case of utilizing very low absorbance values, to reach near-full conversion, over-illumination is required. For an 
initial absorbance of 1 , a $\rho \phi$ value of 3.59 is required to achieve $99.9 \%$ conversion. When the absorbance has a value of only 0.1 , which is common for microreactors, a $\rho \phi$ value of 30.7 is required. These values indicate that 2.59 and 29.7 times more light is introduced in the reactor than could have been avoided by using a more tailored design. This opens up a huge potential for industrial applicability, simply by altering their designs. For cross-current, which is the current state-of-the-art, only suboptimal results are obtained. The most efficient operating point is situated at very high absorbance values. These optimal results are contrary to the current operating reactors, which use low initial absorbance values to achieve homogeneous illumination. Via this current technique, a benchmark score of 0.52 for $A=1$ and 0.035 for $A=0.1$ is acquired, possibly even lower as over-illumination is applied $(\rho \phi \gg 1)$ in the 'state-of-the-art' and, as mentioned above, the use of over-illumination lowers the light utilization efficiency drastically.

\section{Co-current}

Figure 6 Conversion (a), light utilization efficiency (b), Benchmark $\left(\mathrm{X}_{\text {out }}\left(1-\mathrm{t}_{\text {out }}\right)\right)$ (c) and PSTY (d) vs absorbance (A) and light/reagents ratio $(\rho \phi)$, higher values indicate higher conversion and/or higher light efficiency for cocurrent with a plug flow regime. The benchmark's range is [0 1], with 1 being the optimal.

It must be noted that the graphs in Figure 7 look very similar to cross-current irradiation. The main exception compared to cross-current is the applicability range. Reactors typically have a length-overdiameter ratio of more than 10 , typically between $10^{2}$ and $10^{4}{ }^{2}$ Therefore, a different working range in terms of absorbance is obtained, ranging from approximately $A=1$ to complete absorption for longer reactors. In that sense, higher conversion and light efficiency is obtained with those geometries. In terms of the benchmark, due to the geometry constraints of co-current, the optimal working range becomes available: starting from 0.52 at $A=1$ up to more than 0.9 at higher absorbance values at the most optimal $\rho \phi$ value of 1 .

\section{Counter-current}


Figure 7 Conversion (a), light utilization efficiency (b), benchmark $\left(\mathrm{X}_{\text {out }}\left(1-\mathrm{l}_{\text {out }}\right)\right)(\mathrm{c})$ and PSTY (d) vs absorbance $(A)$ and light/reagens ratio $(\rho \phi)$, higher values indicate higher conversion and/or higher light efficiency for cocurrent with a plug flow regime. The benchmark's range is [0 1], with 1 being the optimal.

Counter-current illumination of photochemical reactors shows the best results compared to cross- or cocurrent in terms of conversion at any $(\rho \phi, A)$ combination. A benchmark score of 0.999 is achieved at $\rho \phi=1$ starting from $A=6$, this indicates $99.95 \%$ conversion and $99.95 \%$ light efficiency. Furthermore, this corresponds well with the optimal region in terms of PSTY. Thus, an efficient and productive reactor is achieved at the same time. This result is not achievable with the 'state-of-the-art' or co-current schemes. The reason for this high efficiency is the gradient-linked concentration in the reactor. A gradient is observed from inlet (high concentration) to outlet (low concentration) while the light passes from outlet to inlet. In this configuration, light travels from regions of low to high absorption. Thus at the inlet, the remainder of the light is being absorbed by liquid with a very high absorbance, acting as a "photon sweeper' to collect the last available photons. When looking to counter-current heat transfer, the same effect is observed, via this configuration, the maximum of heat can be transferred. Via co-current, a larger local thermodynamic driving force is observed, but overall this results in lower overall efficiency, this principle is also true for co-current illumination.

\subsubsection{Parameter study - laminar flow}

In the following study, the absorbance $A$ and average photon quantum yield $\rho_{\text {avg }}$ are varied between $[0$ 10] and [0 4], respectively.

\section{Cross-current}

Figure 8 Conversion (a), light utilization efficiency (b), benchmark $\left(\mathrm{X}_{\text {out }}\left(1-\mathrm{t}_{\text {out }}\right)\right)$ (c) and PSTY (d) vs absorbance (A) and light/reagens ratio $(\rho \phi)$, higher values indicate higher conversion and/or higher light efficiency for crosscurrent with a laminar flow regime. The benchmark's range is [0 1], with 1 being the optimal.

The scale of $\rho \phi$ is larger than the one used in plug flow parameter evaluation to accommodate the change in interesting operating region. It must be noted that the optimal operating region, with respect to the benchmark, is no longer present at $\rho \phi=1$, but at a higher value, combined with a high absorbance at the 
cost of a lower PSTY. This physically means that over-illumination of the reactor is required to operate the most efficiently considering the benchmark. The conversion and light efficiency at this optimal region ( $\rho \phi \cong 1.3 \& A=10)$ are $\sim 0.9$ and $\sim 0.65$, respectively, which is not corresponding with the optimal PSTY region. Near-full conversion is only achieved at $\rho \phi$ of 2 and higher. This is related to the maximum local flow rate in the reactor in laminar flow, see Eq. 27. Considering the typical operating region of $A \cong 0.1-1$, suboptimal conditions are typically applied which are comparable to cross-current in plug flow.

\section{Co-current}

Figure 9 Conversion (a), light utilization efficiency (b), benchmark $\left(\mathrm{X}_{\text {out }}\left(1-\mathrm{t}_{\text {out }}\right)\right)(\mathrm{c})$ and PSTY (d) vs absorbance (A) and light/reagents ratio $(\rho \phi)$, higher values indicate higher conversion and/or higher light efficiency for cocurrent with a laminar flow regime. The benchmark's range is [0 1], with 1 being the optimal.

The results and conclusion for co-current in laminar flow are almost identical to cross-current, with the only difference in typical operating region. For co-current, due to the reactor configuration, this is typically $A>1-10$, yielding more favorable conversion and light efficiency.

\section{Counter-current}

Figure 10 Conversion (a), light utilization efficiency (b), benchmark $\left(\mathrm{X}_{\text {out }}\left(1-\mathrm{l}_{\text {out }}\right)\right.$ ) (c) and PSTY (d) vs absorbance $(\mathrm{A})$ and light/reagens ratio $(\rho \phi)$, higher values indicate higher conversion and/or higher light efficiency for co-current with a laminar flow regime. The benchmark's range is [0 1], with 1 being the optimal.

Also for counter-current very similar results can be derived, however, these are much sharper defined. Higher conversions and light efficiencies are possible at lower absorbance values in counter-current operation. Also in this case, over-illumination $(\rho \phi>1)$ is required to yield near-full conversion and to reach the optimal point determined by the benchmark. 


\subsection{Physical and practical implications of non-dimensional groups}

These new models can not only be used for the novel co- and counter-current concepts, but also to simply check which settings are required for optimal conditions with values based on batch experiments. These batch experiments can yield the quantum yield of the reaction, which is the only reaction-specific parameter required to calculate the optimal flow conditions. Another approach, applied by Elliot et al., determines the optimal flow rate experimentally with lacking input parameters. Their approach does not take into account the reactor geometry (i.e. high or low absorbance), nor the correct lamp (i.e. received) light flux. With respect to the latter, they use the light flux based on the electrical grid, not the one actually reaching the reactor surface which is the one required for this scaling procedure. Via the use of the models in this paper, the scale up procedure can be performed more accurately along with some insight provided by the non-dimensional groups. Overall, by changing the absorbance or reactor geometry, deviating results, which are not supported by experiments, will be obtained via the method by Elliott et al. ${ }^{28}$. By using the model described in this work, correct conversion values are obtained.

In this model only two dimensionless groups have to be filled in for scale up or for assessment of the optimal operating conditions; the photon quantum yield $\rho \phi$ and the absorbance $A$. By evaluating the values of these groups, it can be easily distinguished in which regime the reactor is operating. $\rho \phi$ is the most important of the two and expresses the ratio of 'useful' light vs. reagent. If this value is equal to 1 , the flow of 'useful' light $\left[\mathrm{mol} \mathrm{s}^{-1}\right]$ is equal to the reagent flow $\left[\mathrm{mol} \mathrm{s}^{-1}\right]$. A value less than 1 indicates under-illumination and incomplete conversion. A value of more than 1 implies over-illumination and loss in energy efficiency, and possibly formation of side products. Hence, 'useful' is used to account for the quantum yield. At $\rho \phi=1$, more light is introduced, but not all absorbed light is used to form products, hence the introduction of the quantum yield. $\rho \phi$ is fully expressed as:

$$
\rho(\lambda) \phi(\lambda)=\frac{\phi(\lambda) q_{0}(\lambda)}{Q c_{0, A}}(\operatorname{coax})=\frac{\phi(\lambda) G_{0}(\lambda) S}{Q c_{0, A}}(\text { cross })
$$


With $q_{0, \lambda}$ being the light in [einstein $s^{-1}$ ] that reaches the interface of the reactor. This parameter can be determined either via actinometry or, if LEDs are being used, via ray tracing simulation. Both procedures yield the same results. This is the most challenging parameter to obtain. For cross-current, this parameter is expressed as $G_{0, \lambda}$ in [einstein $\mathrm{s}^{-1} \mathrm{~m}^{-2}$ ] and is multiplied by the projected area $S\left[\mathrm{~m}^{2}\right]$ onto which the light is emitted. $Q$ in $\left[\mathrm{m}^{3} \mathrm{~s}^{-1}\right]$ is the flow rate provided by the pump and is easily determined. Lastly, $c_{0, A}$ is the initial concentration of the reagent in $\left[\mathrm{mol} \mathrm{m}^{-3}\right]$ and is also easily determined.

The second important term is the absorbance, the absorbance of component $i$ is expressed as:

$$
A_{i}(\lambda)=\varepsilon_{i}(\lambda) c_{i, 0} \ell
$$

Herein, $\varepsilon_{i}(\lambda)$ is the specific absorbance in $\left[\mathrm{m}^{2} \cdot \mathrm{mol}^{-1}\right]$ and is determined via UV-VIS analysis. The concentration $c_{i, 0}\left[\mathrm{~mol} \mathrm{~m}^{-3}\right]$ is easily determined when preparing the solution. The average path length, $\ell[\mathrm{m}]$, can be determined via actinometry ${ }^{17}$ or via ray tracing simulations. Based on the modelling results, absorbance has to be sufficiently high for decent conversion, see Figure 5 till Figure 10.

When all parameters are known, as well as the approximate flow regime, the $\rho \phi$ value as well as the $A$ value can be calculated and checked against Figure 5 - Figure 10 to estimate the approximate conversion and light efficiency of the reactor. This enables the user to easily check if the absorbance is not too low in the reactor, yielding low efficiencies. A more rigorous approach is to apply the aforementioned equations, relevant to the situation, to a specific problem.

\section{Conclusions}

The new concept of co-axial (co- and counter-current) illumination for photochemical reactors was studied by modelling. By the introduction of a new benchmark, which is the product of the obtained conversion and utilized lamp power, a state-of-the-art model was developed. Within this model, counter-current illumination was determined to be the most efficient for both plug flow and laminar flow, in the case of a non-reversible reaction and non-competitive absorbance. Furthermore, it was established that there is a mismatch in operating region for the current state-of-the-art illumination, i.e. cross-current illumination. 
The quest for homogeneous illumination resulted in the use of milli- to micro-sized reactors coupled with low absorbance values. This operating range $A=\left[\begin{array}{ll}0 & 1\end{array}\right]$ does not correspond to the optimal operating region which is at higher absorbance values. By the nature of co-axial illumination, these higher absorbance values can be accomplished without the creation of radially inhomogeneous zones which are notorious in the current state-of-the-art. ${ }^{7,15}$ Based on these theoretical results, the use of counter-current in plug flow is the most beneficial, yielding the highest conversion and light efficiencies at the lowest absorbance values along with the best PSTY values of all illumination techniques. This indicates that the reactor size can be dramatically decreased compared to co-current for the same conversion and light efficiency. This is true as absorbance is linked to average path length, thus reactor length for co- and counter-current.

From this model, two very important non-dimensional groups were deduced; the quantum photon balance $(\rho \phi)$ and the initial absorbance $(A)$. By determining both, the operating point and efficiency can be determined. This could encourage photochemical reactor designers in selecting the most efficient operating point for their reactor.

\section{Acknowledgments}

Glen Meir thanks FWO-Flanders for a SB PhD fellowship (1SA6219N). Mumin Enis Leblebici thanks FWO-Flanders for a postdoctoral fellowship (39715).

\section{Notation}

$\begin{array}{clc}A & \text { absorbance } & - \\ G_{v} & \text { incident radiation (energy) } & \mathrm{W} \mathrm{m}^{-2} \\ N_{A} & \text { constant of Avogadro } & \mathrm{mol}^{-1} \\ c_{\text {light }} & \text { speed of light } & \mathrm{m} \mathrm{s}^{-1} \\ E & \text { residence time distribution } & -\end{array}$




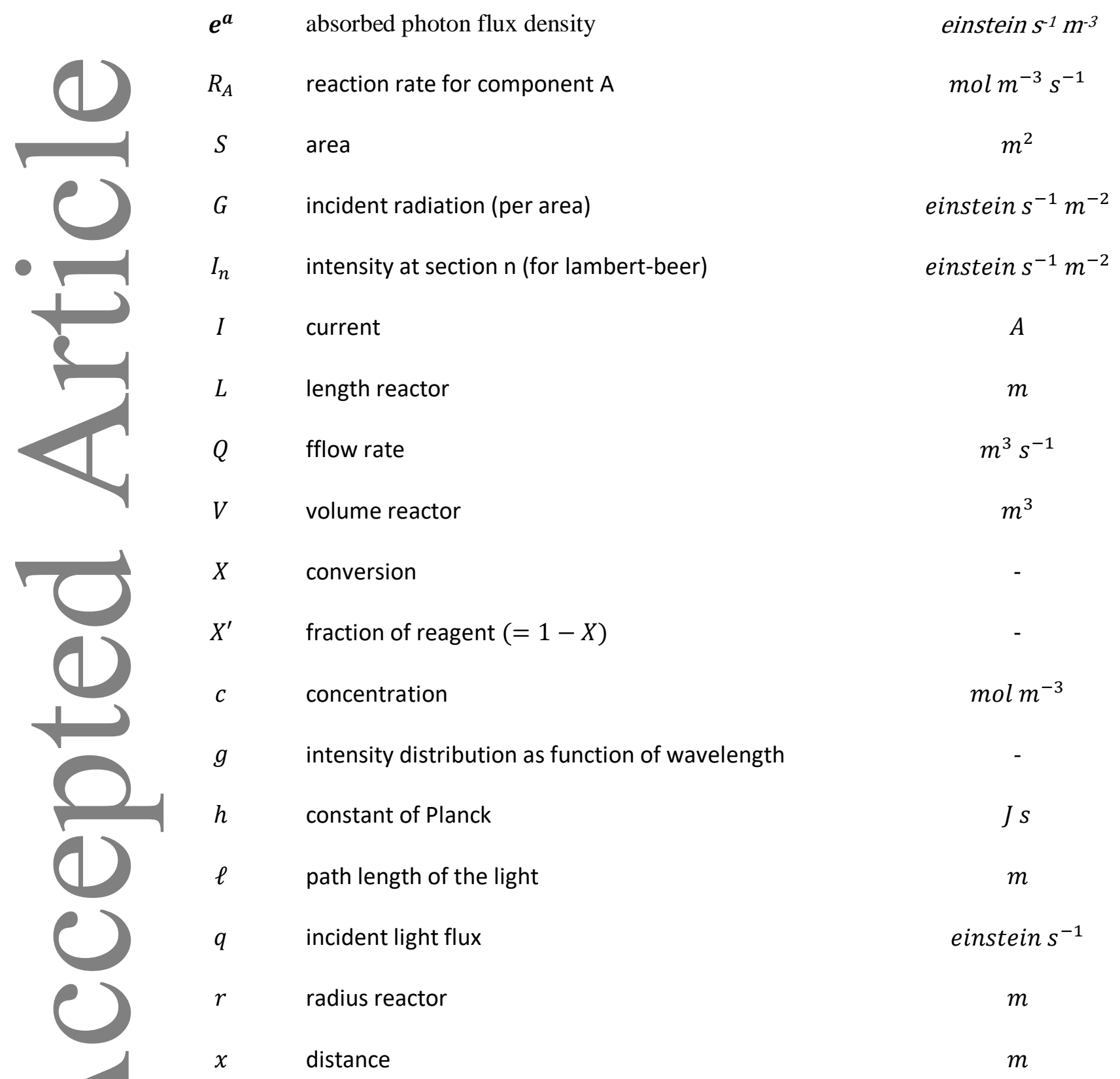

Greek letters

$\Gamma$ correction factor for competitive absorbance $\left(=A_{A} / \sum_{i} A_{i}\right)$ 


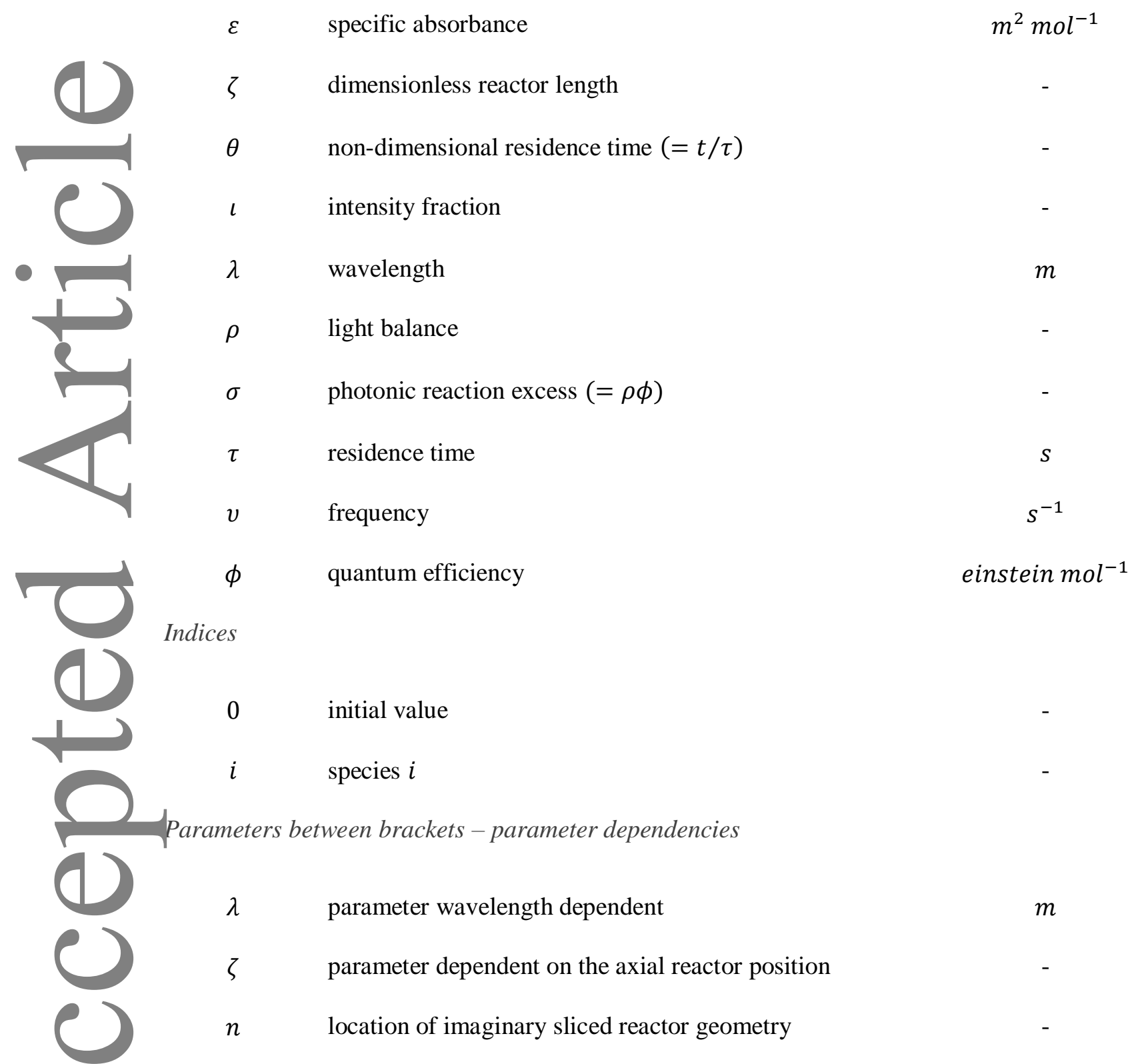

\section{References}

1. Protti S, Dondi D, Fagnoni M, Albini A. Photochemistry in synthesis: where, when, and why. Pure Appl Chem. 2007;79(11):1929-1938. doi:10.1351/pac200779111929

2. Cambié D, Bottecchia C, Straathof NJW, Hessel V, Noël T. Applications of continuous-flow photochemistry in organic synthesis, material science, and water treatment. Chem Rev. 2016;116(17):10276-10341. doi:10.1021/acs.chemrev.5b00707

3. Loubiere K, Oelgemoeller M, Aillet T, et al. Continuous-flow photochemistry: a need for chemical engineering. Chem Eng Pro- cessing Process Intensification, Elsevier. 2016;104:120-132.

4. Plutschack MB, Pieber B, Gilmore K, Seeberger PH. The hitchhiker's guide to flow chemistry. Chem Rev. 2017. doi:10.1021/acs.chemrev.7b00183

5. $\quad$ Noël T. Photochemical Processes in Continuous-Flow Reactors. World Scientific (Europe); 2017. 
doi:10.1142/q0065

6. Sambiagio C, Noël T. Flow Photochemistry: Shine Some Light on Those Tubes! Trends Chem. 2019:1-15. doi:10.1016/j.trechm.2019.09.003

7. Leblebici ME, Stefanidis GD, Van Gerven T. Comparison of photocatalytic space-time yields of 12 reactor designs for wastewater treatment. Chem Eng Process Process Intensif. 2015;97:106111. doi:10.1016/j.cep.2015.09.009

8. Roibu A, Morthala RB, Leblebici ME, Koziej D, Van Gerven T, Kuhn S. Design and characterization of visible-light LED sources for microstructured photoreactors. React Chem Eng. 2018;3(6):849-865. doi:10.1039/c8re00165k

9. Leblebici ME, Van den Bogaert B, Stefanidis GD, Van Gerven T. Efficiency vs. productivity in photoreactors, a case study on photochemical separation of Eu. Chem Eng J. 2017;310:240-248. doi:10.1016/j.cej.2016.10.112

10. Padoin N, Soares C. An explicit correlation for optimal $\mathrm{TiO} 2$ film thickness in immobilized photocatalytic reaction systems. Chem Eng J. 2017;310:381-388. doi:10.1016/j.cej.2016.06.013

11. Baumann M, Baxendale IR. Continuous photochemistry: The flow synthesis of ibuprofen via a photo-Favorskii rearrangement. React Chem Eng. 2016;1:1-10. doi:10.1039/C5RE00037H

12. Dhavale DD, Mali VP, Sudrik SG, Sonawane HR. Media controlled photo-Favorskii type rearrangement of a-chloro acetophenones: synthesis of phenylacetic acids. 1997;53(49):1678916794.

13. Meir G, Leblebici ME, Kuhn S, Van Gerven T. Principles of co-axial illumination for photochemical reactions: Part 2. Model Validation. J Adv Manuf Process. doi:Submitted

14. Alfano M, Cassano E. Radiationfield Modelling in Photoreactors-I. Homogeneous Media. Chem Eng Sci. 1986;41(1955):451-444.

15. Aillet $\mathrm{T}$, Loubière $\mathrm{K}$, Prat $\mathrm{L}$, Dechy-Cabaret $\mathrm{O}$. Impact of the diffusion limitation in microphotoreactors. AIChE J. 2015;61(4):1284-1299. doi:10.1002/aic.14718

16. Elyasi S, Taghipour F. Simulation of UV Photoreactor for Degradation of Chemical Contaminants: Model Development and Evaluation. Environ Sci Technol. 2010;44(6):2056-2063. doi:10.1021/es902391t

17. Roibu A, Fransen S, Leblebici ME, Meir G, Van Gerven T, Kuhn S. An accessible visible-light actinometer for the determination of photon flux and optical pathlength in flow photo microreactors. Sci Rep. 2018;8(1):5421. doi:10.1038/s41598-018-23735-2

18. El Achi N, Bakkour Y, Chausset-Boissarie L, Penhoat M, Rolando C. Rapid and facile chemical actinometric protocol for photo-microfluidic systems using azobenzene and NMR spectroscopy. RSC Adv. 2017;7(47):29815-29820. doi:10.1039/C7RA01237C

19. Maafi M, Brown RG. The kinetic model for $\mathrm{AB}(1 \varphi)$ systems. A closed-form integration of the differential equation with a variable photokinetic factor. J Photochem Photobiol A Chem. 2007;187(2-3):319-324. doi:10.1016/j.jphotochem.2006.10.030

20. Maafi M. The potential of $\mathrm{AB}(1 \Phi)$ systems for direct actinometry. Diarylethenes as successful actinometers for the visible range. Phys Chem Chem Phys. 2010;12(40):13248. doi:10.1039/c0cp00469c

21. Willett KL, Hites RA. Chemical Actinometry: Using o-Nitrobenzaldehyde to measure light 
intensity in photochemical experiments. J Chem Educ. 2000;77(7):900-902. doi:10.1021/ed077p900

22. Zalazar CS, Labas MD, Martín CA, Brandi RJ, Alfano OM, Cassano AE. The extended use of actinometry in the interpretation of photochemical reaction engineering data. Chem Eng J. 2005;109(1):67-81. doi:10.1016/j.cej.2005.03.011

23. Enis Leblebici M, Van den Bogaert B, Stefanidis GD, Van Gerven T. Efficiency Vs. Productivity in Photoreactors, a Case Study on Photochemical Separation of Eu. Chem Eng J. 2016. doi:10.1016/j.cej.2016.10.112

24. Mohajerani M, Mehrvar M, Ein-Mozaffari F. Computational fluid dynamics (CFD) modeling of photochemical reactors. In: Applied Computational Fluid Dynamics. InTech; 2012:155-176. doi: $10.5772 / 26468$

25. Levenspiel O. Chemical Reaction Engineering. Ind Eng Chem Res. 1999;38(11):4140-4143. doi:10.1021/ie990488g

26. Rasmuson A, Andersson B, Olsson L, Andersson R. Mathematical Modeling in Chemical Engineering. 1st ed. New York: Cambridge University Press; 2014.

27. Akul Mehta. Ultraviolet-Visible (UV-Vis) Spectroscopy - Limitations and Deviations of BeerLambert's Law. https://pharmaxchange.info/2012/05/ultraviolet-visible-uv-vis-spectroscopy-limitations-and-deviations-of-beer-lambert-law/. Published 2012. Accessed March 29, 2019.

28. Elliott LD, Knowles JP, Stacey CS, Klauber DJ, Booker-Milburn KI. Using batch reactor results to calculate optimal flow rates for the scale-up of UV photochemical reactions. React Chem Eng. 2018;3(1):86-93. doi:10.1039/C7RE00193B 
Table 1. Full equations and initial conditions (I.C.) to model cross-, co- and counter-current illumination. The equations include absorbance by various chemicals (in green), complex flow regimes via residence time distribution (in red) and polychromatic light e.g. LEDs (in blue).

\begin{tabular}{|c|c|c|}
\hline $\begin{array}{l}\text { Techni- } \\
\text { que }\end{array}$ & Equation(s) & I.C. \\
\hline Cross & $\begin{array}{c}X(\zeta)=\int_{0}^{\infty} \int_{0}^{1} \int_{\lambda_{1}}^{\lambda_{2}} \phi(\lambda) g(\lambda) \iota(\zeta, \lambda) \rho_{A}(\lambda) \theta\left(1-10^{-\left(A_{A}(\lambda)(1-X(\zeta))+A_{i}(\lambda)\right)}\right) \frac{A_{A}(\lambda)(1-X(\zeta))}{A_{A}(\lambda)(1-X(\zeta))+A_{i}(\lambda)} \mathrm{d} \lambda \mathrm{d} \zeta E(\theta) \mathrm{d} \theta \\
\int_{0}^{1} \iota(\zeta, \lambda) \mathrm{d} \zeta=1 \quad \iota(\zeta, \lambda)=1 \text { (homogeneous illumination) }\end{array}$ & $\begin{aligned} X(0) & =0 \\
\iota(0, \lambda) & =\iota_{0}\end{aligned}$ \\
\hline Co & $\begin{array}{c}X(\zeta)=\int_{0}^{\infty} \int_{0}^{1} \int_{\lambda_{1}}^{\lambda_{2}} \phi(\lambda) g(\lambda) \boldsymbol{\iota}(\zeta, \lambda) \rho_{A}(\lambda) \theta \ln (10) A_{A}(\lambda)(1-X(\zeta)) \mathrm{d} \lambda \mathrm{d} \zeta E(\theta) \mathrm{d} \theta \\
\boldsymbol{\iota}(\zeta, \lambda)=\int_{0}^{1}-\boldsymbol{\iota}(\zeta, \lambda) \ln (10)\left(A_{A}(\lambda)(1-X(\zeta))+A_{i}(\lambda)\right) \mathrm{d} \zeta\end{array}$ & $\begin{array}{c}X(0)=0 \\
\boldsymbol{\iota}(\mathbf{0}, \boldsymbol{\lambda})=1\end{array}$ \\
\hline Counter & $\begin{array}{c}X(\zeta)=\int_{0}^{\infty} \int_{0}^{1} \int_{\lambda_{1}}^{\lambda_{2}} \phi(\lambda) g(\lambda) \boldsymbol{\iota}(\zeta, \lambda) \rho_{A}(\lambda) \theta \ln (10) A_{A}(\lambda)(1-X(\zeta)) \mathrm{d} \lambda \mathrm{d} \zeta E(\theta) \mathrm{d} \theta \\
\boldsymbol{\iota}(\zeta, \lambda)=\int_{0}^{1} \boldsymbol{\iota}(\zeta, \lambda) \ln (10)\left(A_{A}(\lambda)(1-X)+A_{i}(\lambda)\right) \mathrm{d} \zeta\end{array}$ & $\begin{array}{c}X(0)=0 \\
\boldsymbol{\iota}(\mathbf{1}, \lambda)=1\end{array}$ \\
\hline
\end{tabular}

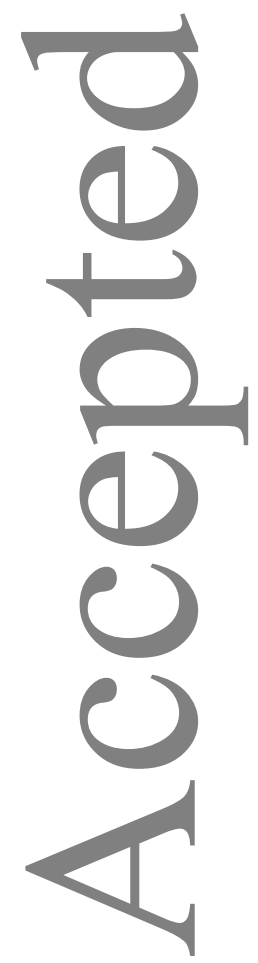

This article is protected by copyright. All rights reserved. 
Table 2. Simplified equations and initial conditions (I.C.) used for parametric evaluation w.r.t. the use of plug flow regime, monochromatic light input and absorbance solemnly by the reagent, leading to the colored terms to be omitted from Table 1.

\begin{tabular}{|c|c|c|}
\hline Technique & Equation(s) & I.C. \\
\hline Cross & $\begin{array}{c}\frac{\mathrm{d} X(\zeta)}{\mathrm{d} \zeta}=\iota(\zeta) \phi \rho_{A}\left(1-10^{-A_{A}(1-X(\zeta))}\right) \\
\int^{1} \iota(\zeta) \mathrm{d} \zeta=1 \quad \iota(\zeta)=1 \text { (homogeneous illumination) }\end{array}$ & $\begin{array}{l}X(0)=0 \\
\iota(0)=\iota_{0}\end{array}$ \\
\hline Co & $\begin{array}{l}\frac{\mathrm{d} X(\zeta)}{\mathrm{d} \zeta}=\iota(\zeta) \phi \rho_{A} \ln (10) A_{A}(1-X(\zeta)) \\
\frac{\mathrm{d} \iota(\zeta)}{\mathrm{d} \zeta}=-\iota(\zeta) \ln (10) A_{A}(1-X(\zeta))\end{array}$ & $\begin{array}{c}X(0)=0 \\
\iota(0)=1\end{array}$ \\
\hline Counter & $\begin{array}{l}\frac{\mathrm{d} X(\zeta)}{\mathrm{d} \zeta}=\iota(\zeta) \phi \rho_{A} \ln (10) A_{A}(1-X(\zeta)) \\
\frac{\mathrm{d} \iota(\zeta)}{\mathrm{d} \zeta}=\iota(\zeta) \ln (10) A_{A}(1-X(\zeta))\end{array}$ & $\begin{array}{c}X(0)=0 \\
\iota(1)=1\end{array}$ \\
\hline
\end{tabular}

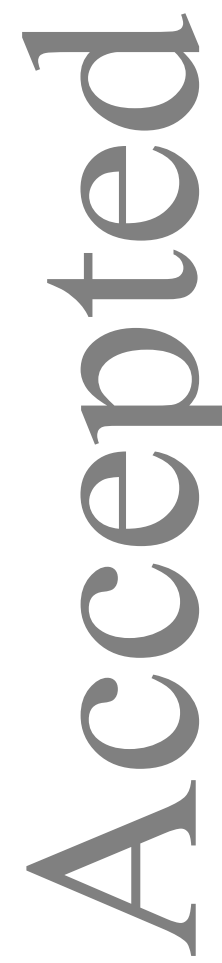

This article is protected by copyright. All rights reserved. 


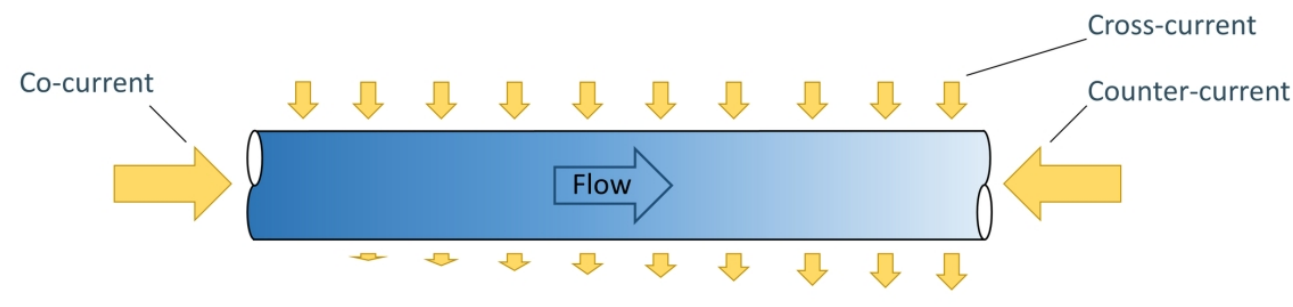

Figure 1: Concept scheme of cross-, co- and counter-current illumination. Note the difference in photon path length by design and the photon losses for cross-current illumination near the end of the reactor. 

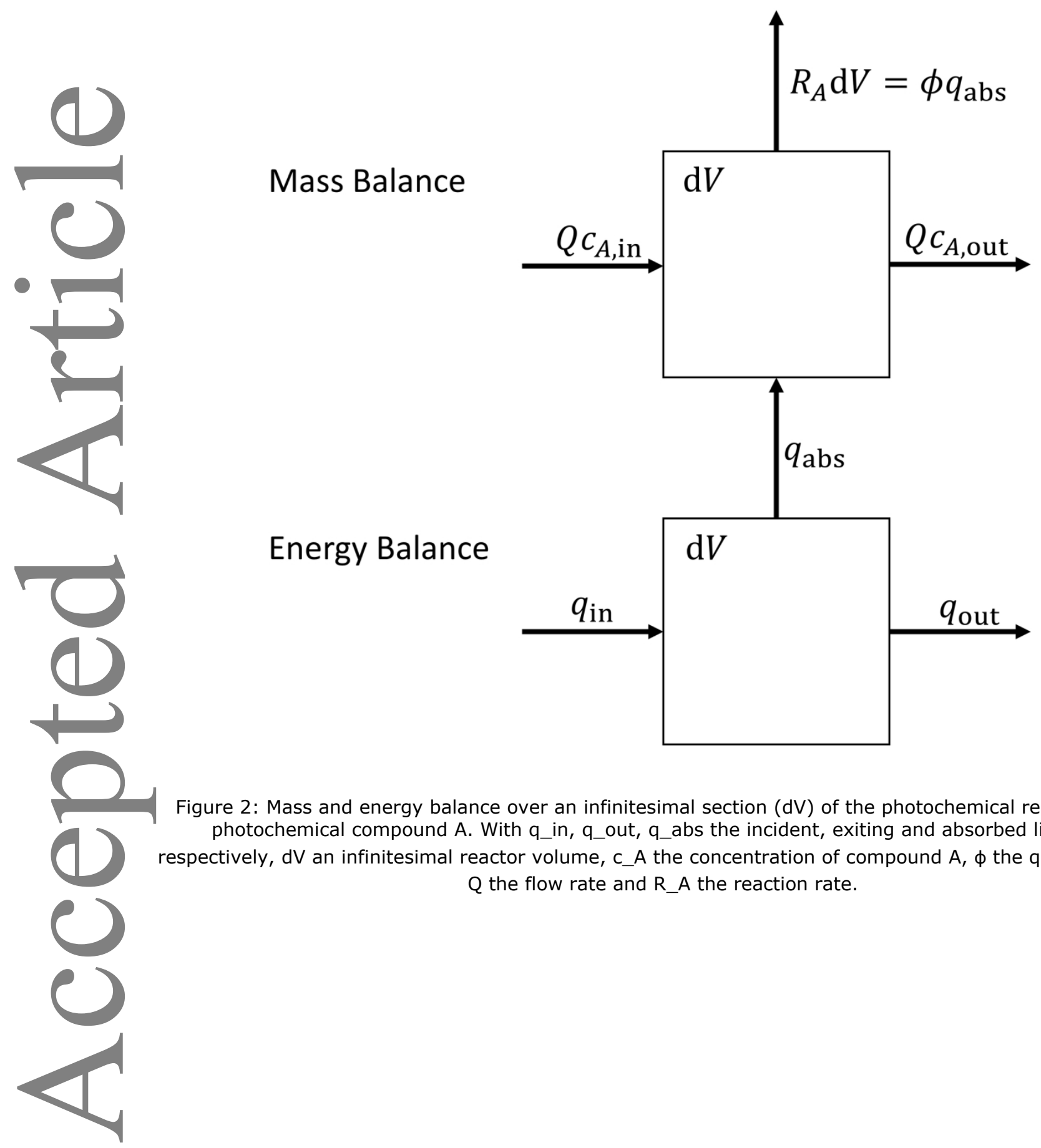

Figure 2: Mass and energy balance over an infinitesimal section (dV) of the photochemical reactor with a photochemical compound A. With q_in, q_out, q_abs the incident, exiting and absorbed light flux respectively, $d V$ an infinitesimal reactor volume, $c \_A$ the concentration of compound $A, \phi$ the quantum yield, $Q$ the flow rate and $R \_A$ the reaction rate. 


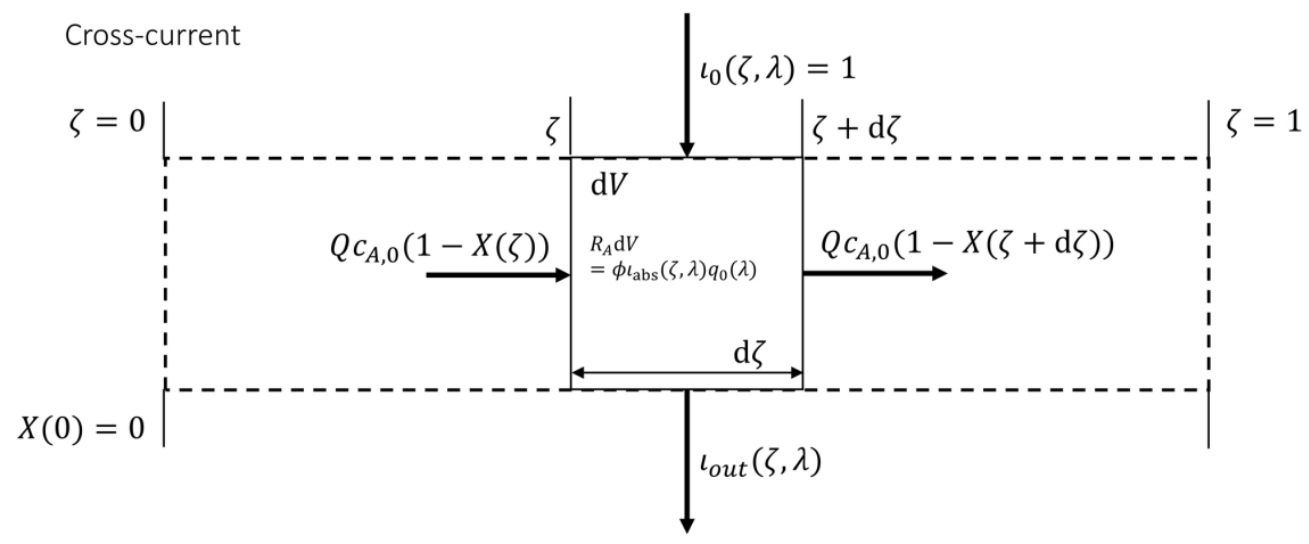

Figure 3: Schematic overview showing the influx and outflux, the boundary conditions for cross-current illumination using a photochemical compound $A$. With $q$ the light flux, I the non-dimensional light flux, $\zeta$ the non-dimensional axial position, $d V$ an infinitesimal reactor volume, $Q$ the flow rate, $c \_A$ the concentration of compound $A, X$ the conversion, $R \_A$ the reaction rate, and $\phi$ the quantum yield. These symbols are the same as in Equation 13. 


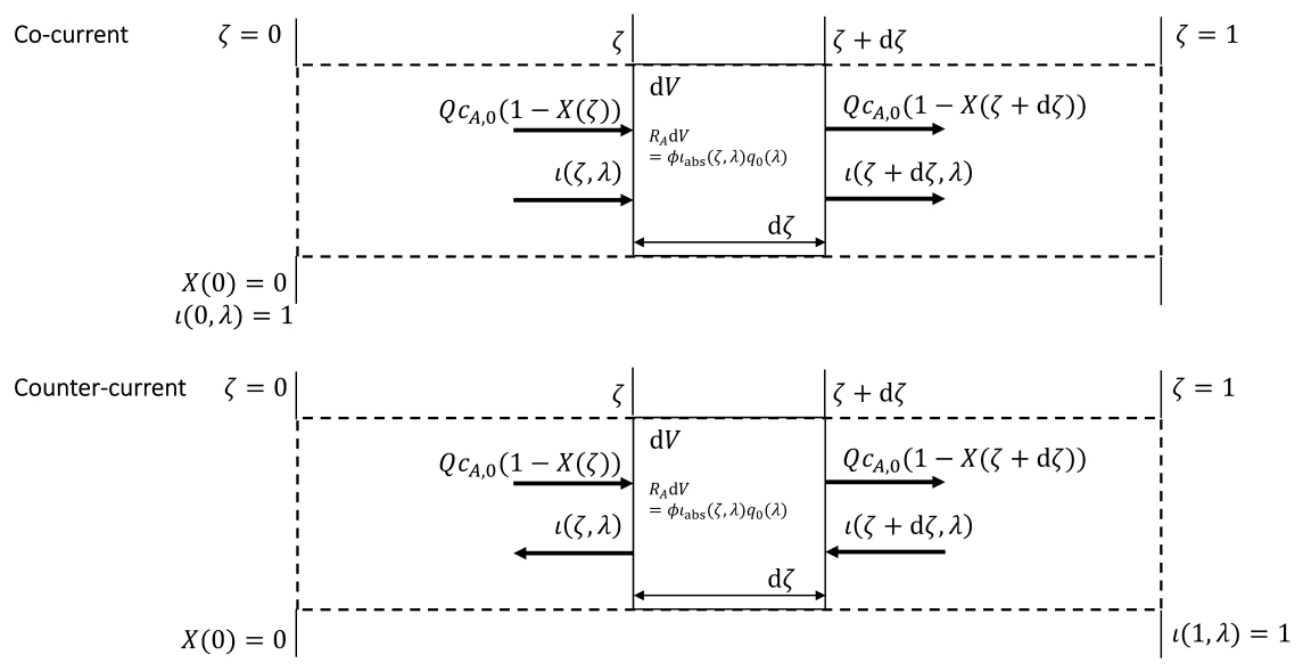

Figure 4: Schematic overview showing the influx and outflux, the boundary conditions for co-current and counter-current illumination using a photochemical compound $A$. With $q$ the light flux, I the non-dimensional light flux, $\zeta$ the non-dimensional axial position, $d V$ an infinitesimal reactor volume, $Q$ the flow rate, $c \_A$ the concentration of compound $A, X$ the conversion, $R \_A$ the reaction rate, and $\phi$ the quantum yield. These symbols are the same as in Equation 19.

This article is protected by copyright. All rights reserved. 

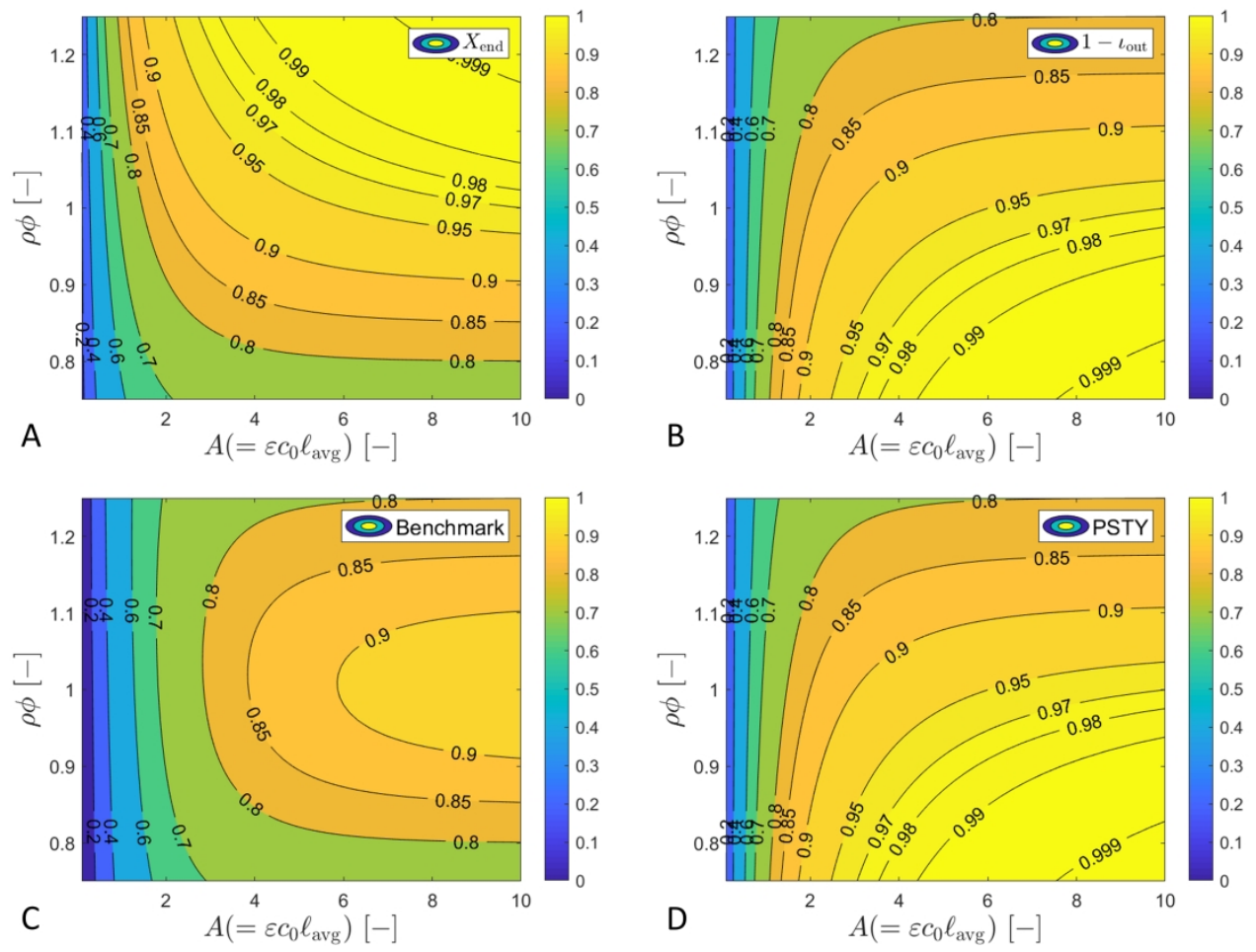

Figure 5 Conversion (a) and light utilization efficiency (b), benchmark (X_out (1-I_out)) (c) and PSTY (d) vs absorbance $(A)$ and light/reagens ratio $(\rho \phi)$, higher values indicate higher conversion and/or higher light efficiency for cross-current with a plug flow regime. The benchmark's range is [0 1], with 1 being the most optimal. 

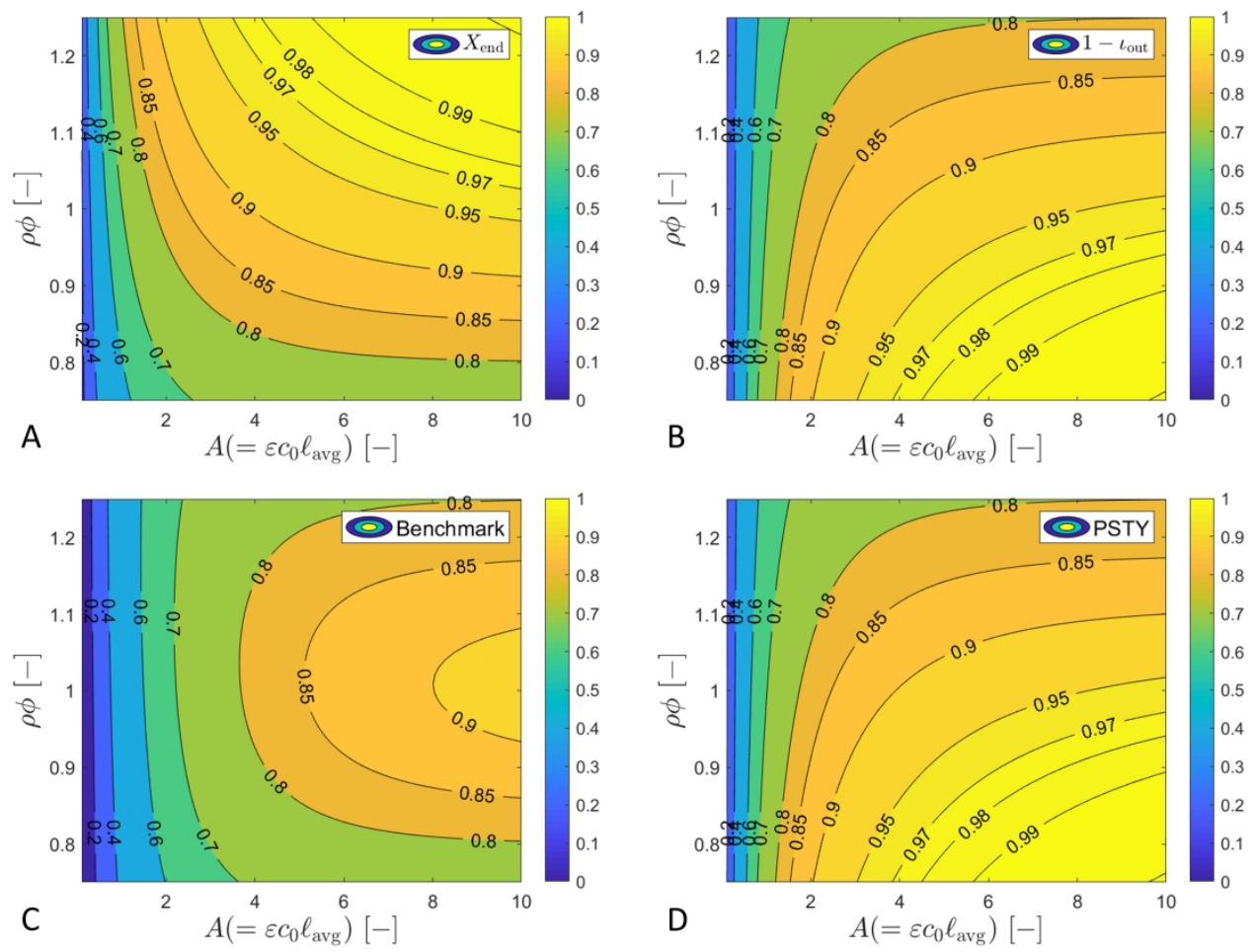

Figure 6 Conversion (a), light utilization efficiency (b), Benchmark (X_out (1-I_out)) (c) and PSTY (d) vs absorbance $(A)$ and light/reagents ratio $(\rho \phi)$, higher values indicate higher conversion and/or higher light efficiency for co-current with a plug flow regime. The benchmark's range is [0 1], with 1 being the optimal. 

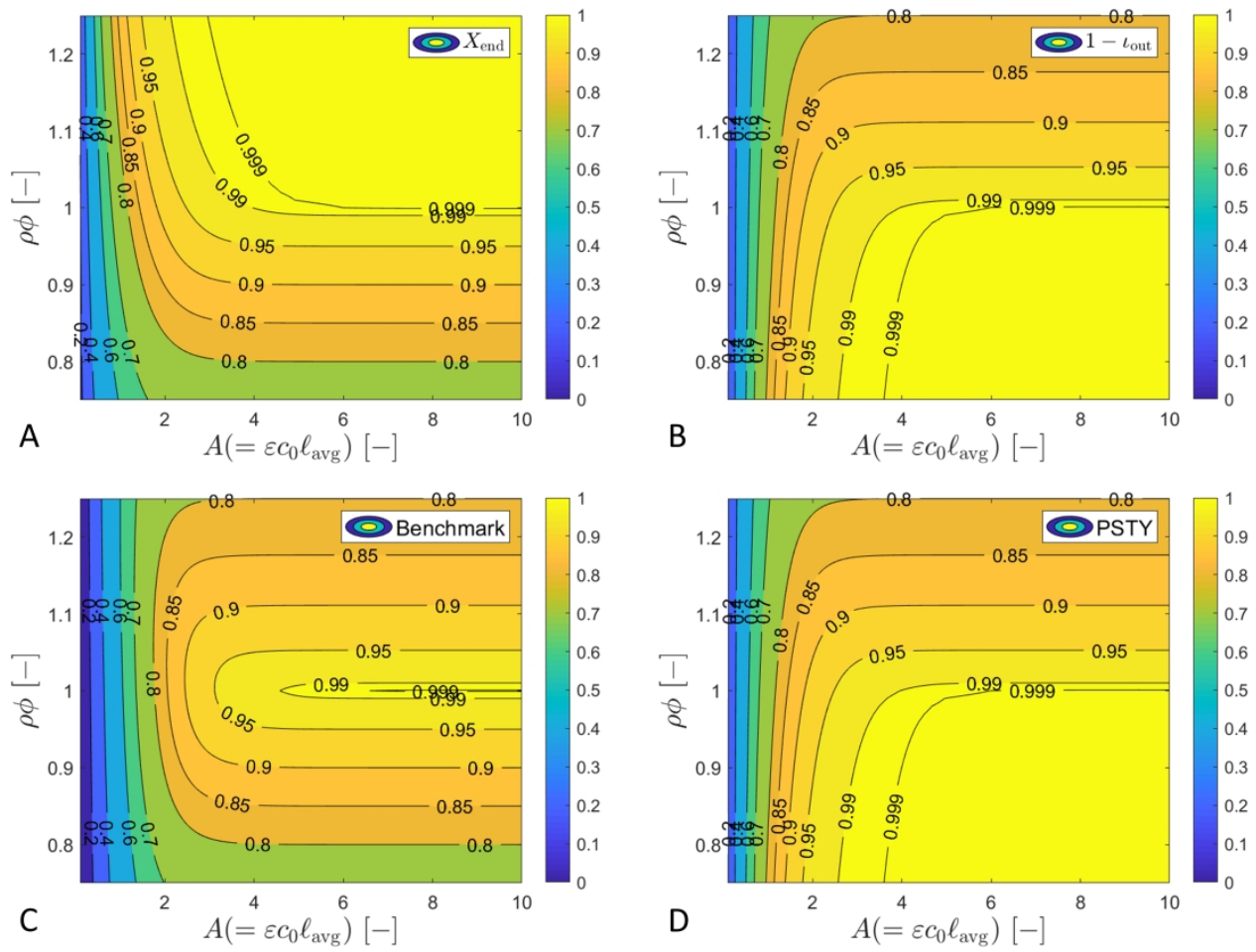

Figure 7 Conversion (a), light utilization efficiency (b), benchmark (X_out (1-I_out)) (c) and PSTY (d) vs absorbance $(A)$ and light/reagens ratio $(\rho \phi)$, higher values indicate higher conversion and/or higher light efficiency for co-current with a plug flow regime. The benchmark's range is [0 1], with 1 being the optimal. 

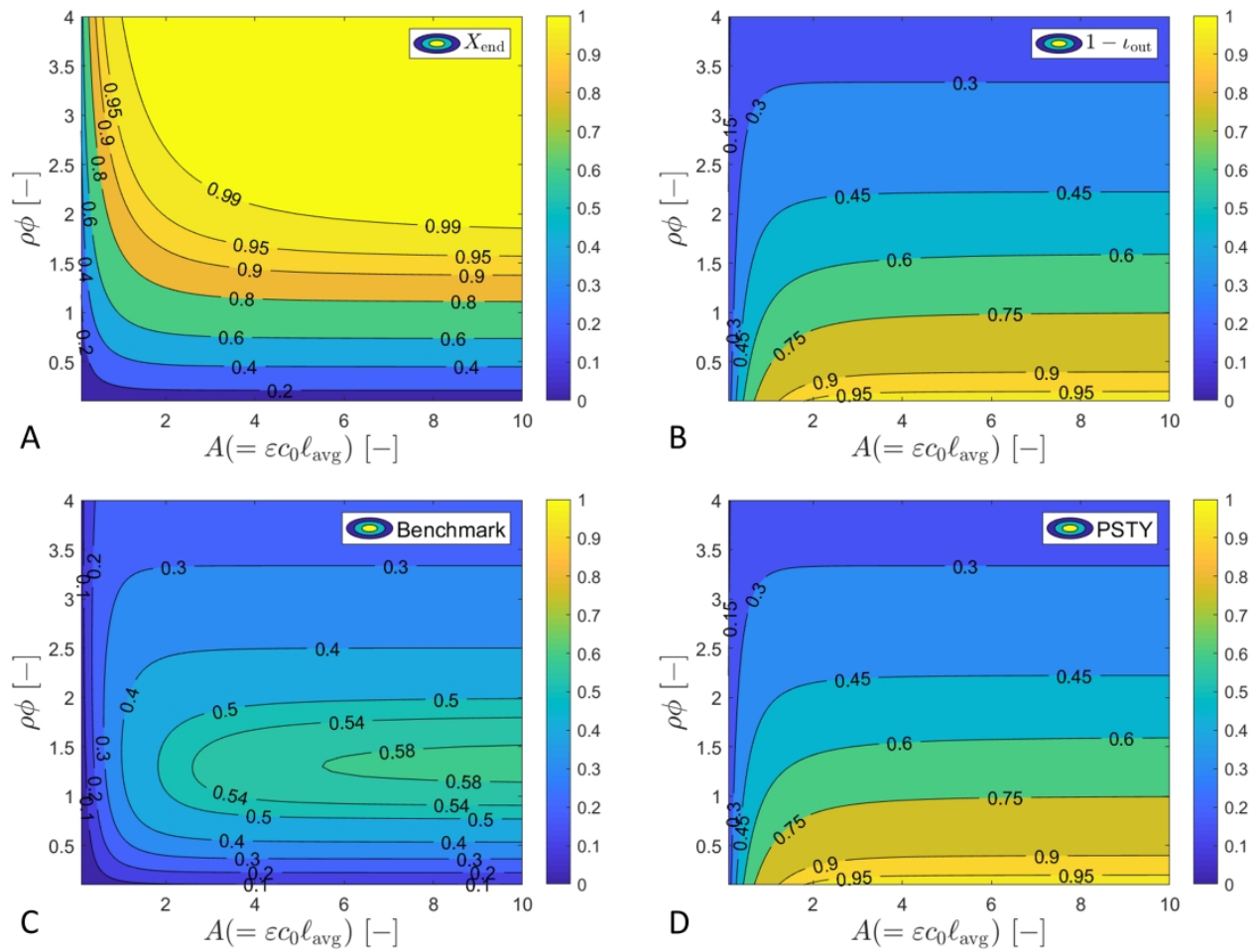

Figure 8 Conversion (a), light utilization efficiency (b), benchmark (X_out (1-I_out)) (c) and PSTY (d) vs absorbance $(A)$ and light/reagens ratio $(\rho \phi)$, higher values indicate higher conversion and/or higher light efficiency for cross-current with a laminar flow regime. The benchmark's range is [0 1], with 1 being the optimal. 

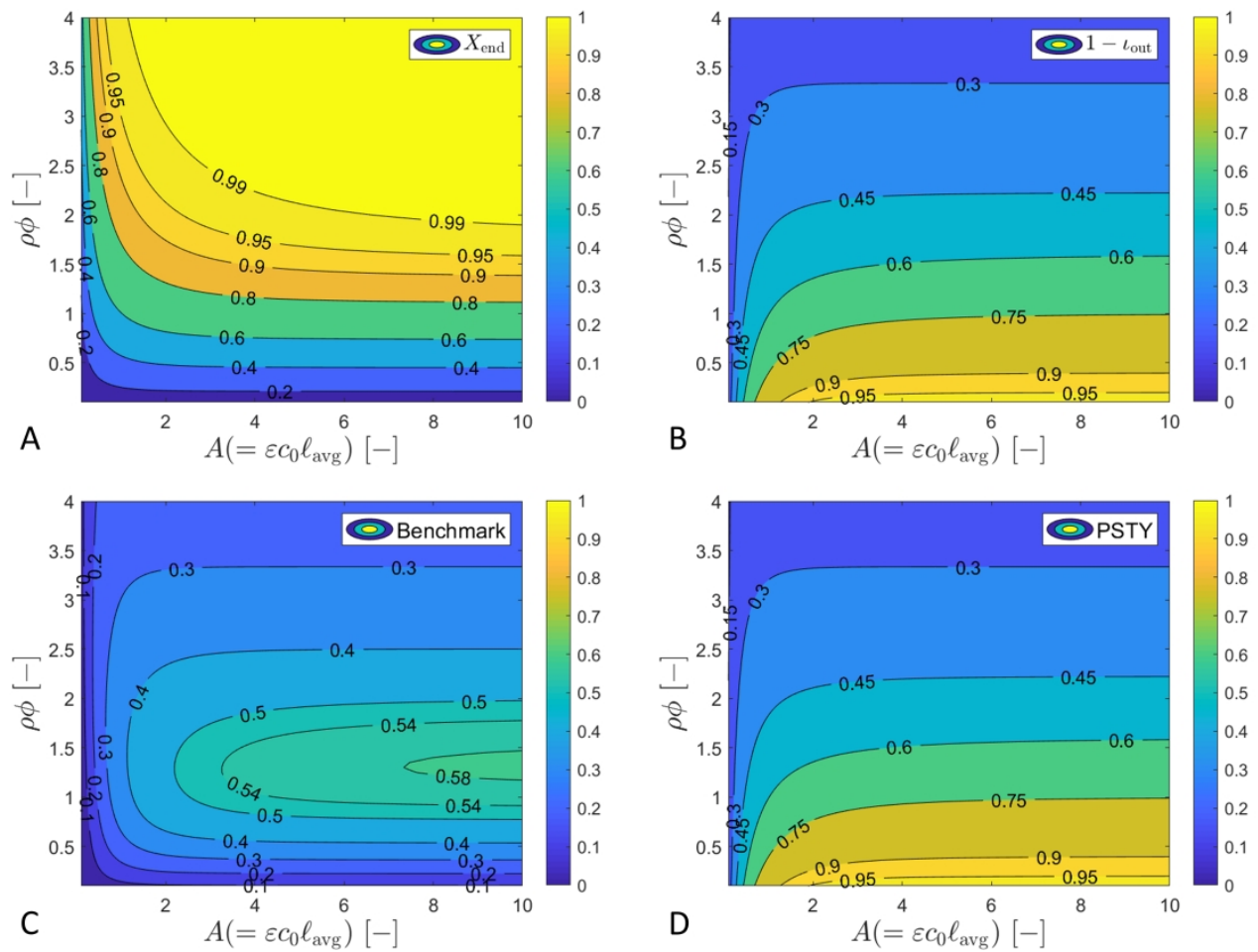

Figure 9 Conversion (a), light utilization efficiency (b), benchmark (X_out (1-I_out)) (c) and PSTY (d) vs absorbance $(A)$ and light/reagents ratio $(\rho \phi)$, higher values indicate higher conversion and/or higher light efficiency for co-current with a laminar flow regime. The benchmark's range is [0 1], with 1 being the optimal. 

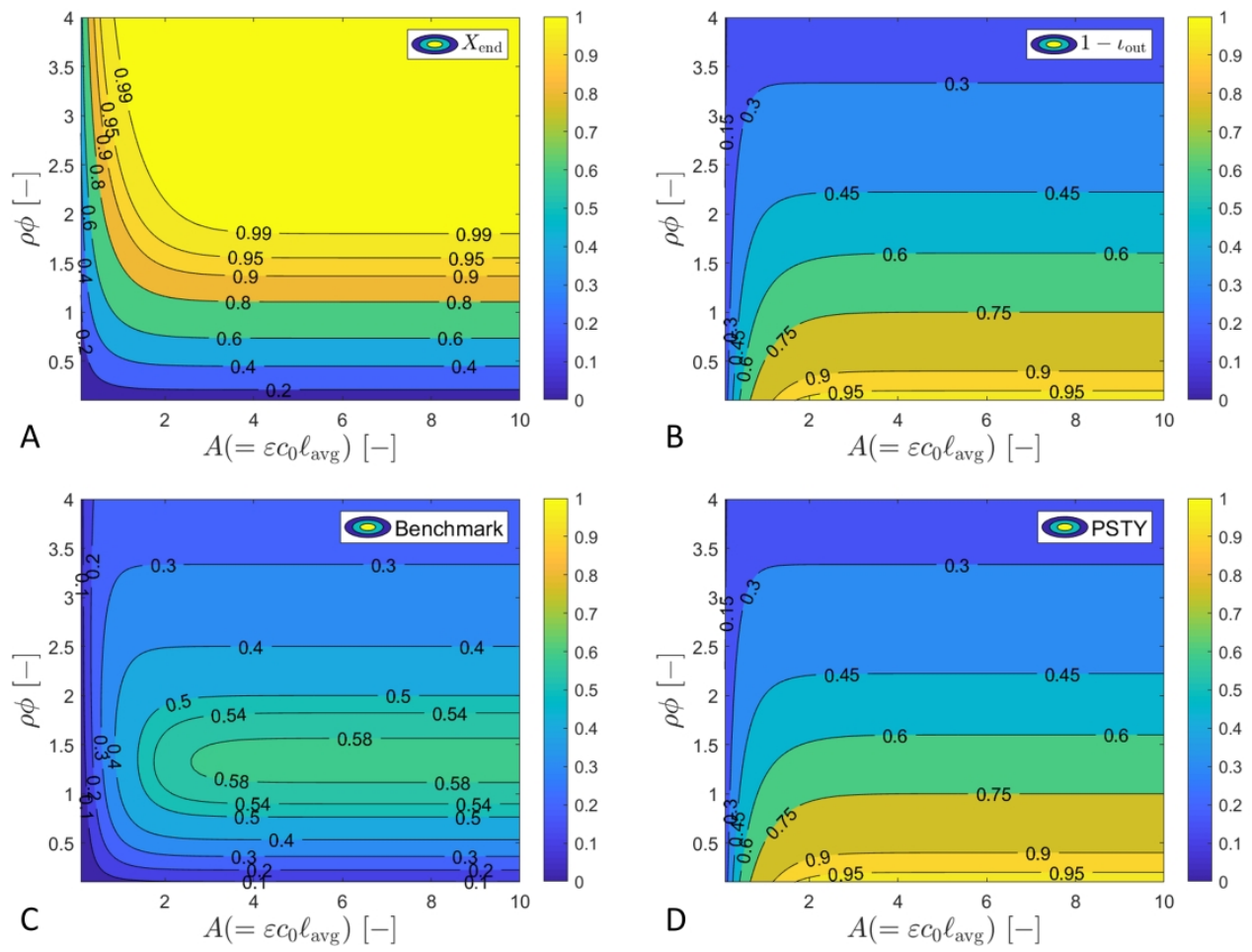

Figure 10 Conversion (a), light utilization efficiency (b), benchmark (X_out (1-I_out)) (c) and PSTY (d) vs absorbance $(A)$ and light/reagens ratio $(\rho \phi)$, higher values indicate higher conversion and/or higher light efficiency for co-current with a laminar flow regime. The benchmark's range is [0 1], with 1 being the optimal. 